\title{
Commonalities and Differences Among Vectorized Beamformers in Electromagnetic Source Imaging
}

\author{
M-X Huang*+, J.J. Shih^, R.R. Lee*+, D.L. Harrington^ ${ }^{\wedge}$, R.J. Thoma*\%, M.P. Weisend*+, \\ F. Hanlon\#, K.M. Paulson\%, T. Li*+, K. Martin*, G.A. Miller\$, and J.M. Canive*\%\&
}

\begin{abstract}
Summary: A number of beamformers have been introduced to localize neuronal activity using magnetoencephalography (MEG) and electroencephalography (EEG). However, currently available information about the major aspects of existing beamformers is incomplete. In the present study, detailed analyses are performed to study the commonalities and differences among vectorized versions of existing beamformers in both theory and practice. In addition, a novel beamformer based on higher-order covariance analysis is introduced. Theoretical formulas are provided on all major aspects of each beamformer; to examine their performance, computer simulations with different levels of correlation and signal-to-noise ratio are studied. Then, an empirical data set of human MEG median-nerve responses with a large number of neuronal generators is analyzed using the different beamformers. The results show substantial differences among existing MEG/EEG beamformers in their ways of describing the spatial map of neuronal activity. Differences in performance are observed among existing beamformers in terms of their spatial resolution, false-positive background activity, and robustness to highly correlated signals. Superior performance is obtained using our novel beamformer with higher-order covariance analysis in simulated data. Excellent agreement is also found between the results of our beamformer and the known neurophysiology of the median-nerve MEG response.
\end{abstract}

Key words: Beamformer; MEG; Dipole; Somatosensory; Median nerve; Inverse problem.

\section{Introduction}

Recently, a variety of beamformer approaches have been introduced to study neuronal activity using electroencephalography (EEG) or magnetoencephalography (MEG) (Van Veen et al. 1997; Robinson and Vrba 1999;

${ }^{*}$ Center for Functional Brain Imaging, $\sim$ Research Service, ${ }^{\%}$ Psychiatry Service, New Mexico VA Health Care System, Albuquerque, NM, USA.

${ }^{+}$Department of Radiology, ${ }^{\wedge}$ Department of Neurology, " Department of Psychology, and \&Department of Psychiatry, University of New Mexico Health Sciences Center, Albuquerque, NM, USA.

$\$$ Biomedical Imaging Center and Departments of Psychology and Psychiatry, University of Illinois, Urbana, IL, USA.

Accepted for publication: November 13, 2003.

This work was supported in part by a VA Merit Review Grant from the US Department of Veterans Affairs, an NIH/National Center for Research Resources grant (P20-RR15636-01), an NIH/National Institute of Mental Health grant (R01-MH65304-01), and a RAC grant from the University of New Mexico.

Correspondence and reprint requests should be addressed to Ming-Xiong Huang, Ph.D., Center for Functional Brain Imaging, Building 49 (114M), New Mexico VA Health Care System, 1501 San Pedro Drive, SE, Albuquerque, New Mexico, 87108, USA.

Tel: (505) 265-1711 ext 4855

Fax: (505) 256-5708

E-mail: mhuang@unm.edu

Copyright (C) 2004 Human Sciences Press, Inc.
Gross and Ioannides 1999; Sekihara et al. 2001; Gross et al. 2001; Barnes and Hillebrand 2003). A beamformer in EEG/MEG is a set of spatial filters based on the EEG/MEG lead fields, the covariance matrix of the signal evaluated for an epoch wherein the number of time points is several times as many as the number of measurement channels (Van Veen et al. 1997), and sometimes the covariance matrix of the noise. The basic principle of beamformer design is to allow the neuronal signal of interest to pass through in certain source location(s) and orientation(s), called pass-band(s), while suppressing noise or unwanted signal in other source location(s) or orientation(s), called stop-band(s). The beamformer approaches were originally developed as signal processing methods to detect signals using receptor arrays in acoustic and radio signals (for detailed review, see Van Veen and Buckley 1988). All existing beamformers in the EEG and MEG literature are narrow-passing-band beamformers, in which either the entire brain volume or just the cortical surface is divided into a grid of dipoles, and at each grid node, the beamformer allows signal from that node to pass and suppress signal/noise from other nodes.

Beamformer approaches have certain advantages over traditional multiple dipole fitting. Unlike multiple dipole fitting where the number of dipoles has to be determined in advance (Huang et al. 1998), the beamformer requires no a priori assumptions about the number of 
sources to model (Van Veen et al. 1997). In addition, the dipole approach which depends on assumption of a point source may not be able to accurately describe the underlying neuronal sources with large extents, whereas the beamformer has the capability to handle such sources (Van Veen et al. 1997). The beamformer technique also has advantages over minimum-norm (L2 and L1 norm) solutions. Minimum-norm solutions are often smeared, and their spatial resolution can be low for focal point-like neuronal sources, while the beamformer can model the point sources down to the size of the mesh grid (Barnes and Hillebrand 2003) under favorable signal-to-noise ratios (SNR). Minimum-norm solutions also tend to be biased toward superficial sources, thus weighting is often required to handle deep sources. In contrast, beamformers can easily handle both superficial and deep sources. Furthermore, statistical tests are usually difficult for both dipole fitting and minimum-norm solutions, whereas a variety of statistical analyses can be easily implemented using beamformer approaches (Barnes and Hillebrand 2003; Gross et al. 2001; Robinson and Vrba 1999). One potential problem with beamformer analyses is that they are unable to distinguish two sources if their time-courses are 100\% correlated. Although Van Veen and colleagues (1997) showed that their beamformer was able to separate two sources whose time-courses correlated $50 \%$, it is unknown how robust other beamformers are in handling correlated sources.

For any beamformer approach, there are four important characteristics (and associated formulas): 1) beamformer coefficients or weights; 2) neuronal source time-courses; 3) neuronal activity indexes, a term for describing the spatial map of the underlying neuronal activity (Van Veen et al. 1997) for the entire time interval in which the covariance matrix is calculated; and 4) neuronal activity indexes for each time point. However, in the literature only a subset of these four characteristics are typically described. Consequently, the commonalities and differences among various beamformer approaches are not clearly understood. For example, the "minimum variance beamformers" of Van Veen and colleagues (1997) and of Robinson and Vrba (1999) are assumed to be the same approach (Sekihara et al. 2001; Barnes and Hillebrand 2003), except that one uses a regularization technique (Robinson and Vrba 1999), and the other does not (Van Veen et al. 1997). However, as we will demonstrate, this is not the case. Likewise, Sekihara and colleagues (2001) derived a beamformer claimed to be based on the beamformer technique of Borgiotti and Kaplan (1979), and claimed to be very different from the well-known minimum variance beamformer. Although the simulations of Sekihara and colleagues (2001) appeared to support this statement, an examination of the original equations from Borgiotti and Kaplan (1979) clearly shows that the Borgiotti-Kaplan beamformer used exactly the same design as the minimum variance beamformer.

The goal of the present study is to demonstrate at both theoretical and practical levels the commonalities and differences among all existing beamformers in EEG and MEG. In doing so, we will provide comprehensive sets of formulas covering all four important aspects of each beamformer. This will provide users with a complete picture about the strengths and weaknesses of each beamformer when applied to empirical electromagnetic data. As we will show later, significant differences exist among a variety of linearly-constrained beamformers, particularly in terms of obtaining the spatial map of the neuronal activity indexes.

The present study begins by providing the theoretical foundations for each beamformer in the Methods section. Detailed formulas are given regarding each beamformer's weights, source time-courses, and neuronal activity indexes both for each time point and the entire interval in which the data covariance matrix is calculated. Next, we introduce the theoretical foundation of a novel beamformer based on higher-order covariance analysis. In the Results section, computer simulation results are presented that examine the spatial resolution of the beamformers at different SNRs for two dipole sources with nearly uncorrelated time-courses, and for two dipole sources with time-courses that are correlated to different degrees. Finally, the performances of the beamformers are compared using measured data from an evoked median-nerve MEG response from a normal human subject. This task is particularly appropriate because median-nerve stimulation has been routinely used in humans to study the somatosensory system. Consequently, we have an excellent understanding of the underlying neuronal systems, which allowed us to predict with confidence where sources should be found. However, except for one EEG study (Van Drongelen et al. 1996), median-nerve responses have not been analyzed using a beamformer approach. This may be due to the relatively large number of neuronal sources activated in a relatively short period of time by the median-nerve stimulation with typical repetition rates, which challenges covariance-based analysis techniques such as beamformer. For example, previous neurophysiology studies (including MEG) have shown that strong stimulation of the peripheral nerve can activate: 1) contralateral primary somatosensory area (SI) with a first component around $20 \mathrm{~ms}$ post-stimulus in humans (Wood et al. 1985; Hari et al. 1993; Forss et al. 1994; Kawamura et al. 1996; Mauguiere et al. 1997a; Mauguiere et al. 1997b; Forss and Jousmaki 1998; Jousmaki and Forss 1998; Hari and Forss 1999; Huang et al. 2000); 2) contralateral primary motor area (MI) with a first component around 20-30 ms post-stimulus in humans (Rosen and Asanuma 1972; Lemon and Porter 1976; Jones et al. 1978, 1979; Wong et al. 1978; Lemon van der Burg 1979; Lemon 
1981; Davidoff 1990; Baldissera and Leocani 1995; Kawamura et al. 1996; Spiegel et al. 1999; Huang et al. 2000); 3) contralateral superior parietal area (Jones et al. 1978, 1979; Forss et al. 1994; Boakye et al. 2000; McGlone et al. 2002; Waberski et al. 2002); 4) supplementary motor area (SMA) (Urbano et al. 1997; Boakye et al. 2000; Barba et al. 2001); 5) premotor area (Park and Del Toro 1995; Mauguiere et al. 1997b; Bergeron and Braddom 1998); and 6) bilateral secondary somatosensory areas (SII) (Hari et al. 1993; Forss and Jousmaki 1998; Hari and Forss 1999; Fujiwara et al. 2002; Simoes et al. 2003). This type of challenge is thus especially valuable for evaluating the limitations and relative performance of various beamformer strategies in real-world data analysis.

\section{Methods}

\section{Vectorized Linearly-Constrained Minimum-} Variance Beamformer

The electric potential measured by EEG or magnetic fields measured by MEG for an interval of time can be expressed in an $M \times N$ data matrix:

$$
\mathbf{B}(t)=\left[\mathbf{b}\left(t_{1}\right), \mathbf{b}\left(t_{2}\right), \ldots, \mathbf{b}\left(t_{N}\right)\right]
$$

where $N$ is the number of time samples in the data and $\mathbf{b}\left(t_{i}\right)$ is a $M \times 1$ vector containing the electric potentials or magnetic fields at $M$ sensor sites for a specific time point $t_{i}$. The $\mathbf{b}\left(t_{i}\right)$ can be expressed in terms of the lead-fields, active dipole time-courses, and noise:

$$
\mathbf{B}(t)=\mathbf{L Q}(t)+\text { Noise }(t)
$$

where $\mathbf{L}$ is, in general, an $M \times 3 P$ lead field matrix for $P$ dipole locations. Each column of $\mathbf{L}$ is called a lead-field vector representing the electric potentials/magnetic fields at $M$ sensors due to the unit dipole moment at a dipole location pointing at either $x, y$, or $z$ principal direction using the Cartesian coordinate system (or $\rho, \theta, \phi$ direction using the spherical coordinate system). In the special case of the MEG spherical head model, the radial component of the dipole moment has no contribution to the MEG measurements, and the lead-field matrix then becomes an $M \times 2 P$ matrix. The $\mathbf{Q}(t)$ in equation 2 is a $3 P \times N$ dipole moment matrix, and Noise $(t)$ is the noise matrix. Each row of $\mathbf{Q}(t)$, namely $\mathbf{q}(t)$, represents the dipole time-course at a specific dipole location and orientation. In general, we can partition $\mathbf{L}$ into $P$ sub-arrays:

$$
\mathbf{L}=\left[\mathbf{L}_{1}, \mathbf{L}_{2}, \ldots, \mathbf{L}_{P}\right]
$$

where each $M \times 3$ sub-array $\mathbf{L}_{i}=\left[\mathbf{1}_{x}, \mathbf{1}_{y}, \mathbf{1}_{z}\right]_{i}$ represents the lead-fields at a dipole location $i$ for $x, y$, and $z$ principal dipole orientations. Note that the Cartesian coordinate system is used here and in other equations for convenience. All equations still hold if one uses the spherical coordinate system by simply replacing $x, y, z$ with $\rho, \theta, \phi$.

Traditionally, beamformer approaches in EEG/MEG only contain $P$ beamformers, one for each dipole location, by assuming that the dipole orientation is either known in advance or can be obtained through an extra optimization step (Sekihara et al. 1999; Barnes and Hillebrand 2003; Vrba and Robinson 2002). However, such an assumption is not mandatory and can be replaced by designing separate beamformers for each individual principal dipole orientation using the vectorized beamformer approaches (Sekihara et al. 2001; Van Veen et al. 1997). Such a vectorized beamformer design contains $3 P$ beamformers, three for each dipole location corresponding to the three principal dipole orientations. Each beamformer uses a linear combination of the measured signals $\mathbf{B}(t)$ to estimate the dipole time-course at each dipole location and orientation:

$$
\widehat{\mathbf{q}}_{k}(t)=\mathbf{w}_{k}^{T} \mathbf{B}(t), \text { for } k=1,2, \ldots, 3 P
$$

where $\mathbf{w}_{k}$ is a $M \times 1$ beamformer coefficient vector (or weights) and $\mathbf{q}_{k}^{\wedge}(t)$ is an $1 \times N$ estimated dipole time-course vector in one of the principal directions. The estimated total power at each dipole location and orientation can be written as:

$$
S_{k}=\mathbf{w}_{k}^{T} \mathbf{C} \mathbf{w}_{k}
$$

where $\mathrm{C}$ is the $M \times M$ covariance matrix calculated over a time window containing $N$ time samples:

$$
\mathbf{C}=\left\langle(\mathbf{b}(t)-\langle\mathbf{b}(t)\rangle)(\mathbf{b}(t)-\langle\mathbf{b}(t)\rangle)^{T}\right\rangle
$$

where $<\bullet>$ indicates the ensemble average across $N$ time samples.

The problem of solving vectorized linearly-constrained minimum variance beamformer can be expressed as:

$$
\min \left\{S_{k}\right\} \text { subject to: } \mathbf{w}_{k}^{T} \mathbf{1}_{k}=1 \text { for } k=1,2, \ldots, 3 P \text {. }
$$

In a sense, the vectorized linearly-constrained minimum-variance beamformer is a set of $3 P$ spatial filters, which allow the signal to pass for designated dipole location and orientation and stop the signal if the dipole location or orientation is different from the designated location or orientation. The solution to equation 7 can be easily obtained using the Lagrange multiplier method: 


$$
\mathbf{w}_{k}=\mathbf{C}^{-1} \mathbf{l}_{k}\left(\mathbf{l}_{k}^{T} \mathbf{C}^{-1} \mathbf{l}_{k}\right)^{-1} \text { for } k=1,2, \ldots, 3 P
$$

One can then substitute equation 8 into equation 4 and obtain the dipole time-course for each location and principal orientation. In general, the designing phase of the vectorized linearly-constrained minimum-variance beamformer ends when the beamformer coefficients are provided by equation 8 . However, as shown in the next subsection, substantial differences start to show up among different beamformers when estimating the spatial distribution of the underlying neuronal sources, also called neuronal activity indexes (Van Veen et al. 1997).

By substituting equation 8 into equation 5 , one can also obtain the source power for the entire time window in which the covariance matrix is estimated:

$$
S_{k}=\mathbf{w}_{k}^{T} \mathbf{C} \mathbf{w}_{k}=\left(\mathbf{1}_{k}^{T} \mathbf{C}^{-1} \mathbf{1}_{k}\right)^{-1} \text { for } k=1,2, \ldots, P
$$

However, one cannot use equation 9 directly to describe the spatial distribution of the underlying neuronal activity. This is because equation 9 shows erroneously large values near the center of the head and the noise is non-uniformly distributed throughout the source space (Van Veen et al. 1997; Robinson and Vrba 1999; Sekihara et al. 2001). Another problem with equation 9 is that for each dipole location, there are three estimations of the source power corresponding to $x, y$, and $z$ dipole orientations, while usually the user is interested in one combined measure for each dipole site.

\section{Vectorized Type 1 Beamformer}

To deal with the above problems, Van Veen and colleagues (1997) proposed the following measure of neuronal activity index for the entire interval in which the covariance matrix is evaluated:

$$
\begin{aligned}
R_{\text {type } 1}= & \frac{\operatorname{tr}\left\{\left(\mathbf{L}_{i}^{T} \mathbf{C}^{-1} \mathbf{L}_{i}\right)^{-1}\right\}}{\operatorname{tr}\left\{\left(\mathbf{L}_{i}^{T} \Sigma^{-1} \mathbf{L}_{i}\right)^{-1}\right\}} \\
& =\frac{\left(\mathbf{1}_{x}^{T} \mathbf{C}^{-1} \mathbf{1}_{x}\right)_{i}^{-1}+\left(\mathbf{1}_{y}^{T} \mathbf{C}^{-1} \mathbf{1}_{y}\right)_{i}^{-1}+\left(\mathbf{1}_{z}^{T} \mathbf{C}^{-1} \mathbf{1}_{z}\right)_{i}^{-1}}{\left(\mathbf{1}_{x}^{T} \Sigma^{-1} \mathbf{l}_{x}\right)_{i}^{-1}+\left(\mathbf{l}_{y}^{T} \Sigma^{-1} \mathbf{l}_{y}\right)_{i}^{-1}+\left(\mathbf{l}_{z}^{T} \Sigma^{-1} \mathbf{1}_{z}\right)_{i}^{-1}}
\end{aligned}
$$$$
\text { for } i=1,2, \ldots, P
$$

Recall that $\mathbf{L}_{i}=\left[\mathbf{1}_{x}, \mathbf{1}_{y}, \mathbf{1}_{z}\right]_{i}$ (see equation 3 ) is the $M \times 3$ sub-array lead-fields at for $x, y$, and $z$ dipole orientations for each dipole location $i, \Sigma$ is the noise covariance matrix, and $\operatorname{tr}\{\bullet\}$ represents the trace operation over the three principal dipole orientations. Note that the index $i$ goes from 1 to $P$, not $3 P$. In a sense, equation 10 takes the total power among the principal orientations and then divides it by the total noise power among these orientations. The dipole time-courses can be obtained from equation 4 and equation 8 .

An important piece of information not directly available from Van Veen and colleagues (1997) is the expression of neuronal activity index for any time point where the measured electric potentials/magnetic fields are $\mathbf{b}(t)$. This can be easily derived by calculating the power of the dipole moment at each time point and dividing that power by the same denominator as in equation 10:

$$
r_{\text {type } \_1}(t)=\frac{\operatorname{tr}\left\{\mathbf{W}_{i}^{T} \mathbf{b}(t) \mathbf{b}(t)^{T} \mathbf{W}_{i}\right\}}{\operatorname{tr}\left\{\left(\mathbf{L}_{i}^{T} \Sigma^{-1} \mathbf{L}_{i}\right)^{-1}\right\}} \text { for } i=1,2, \ldots, P
$$

where $\mathbf{W}_{i}=\left[\mathbf{w}_{x}, \mathbf{w}_{y}, \mathbf{w}_{z}\right]_{i}$ is the $M \times 3$ matrix containing the coefficients of the beamformer at all three principal directions for each dipole location $i$. Again, the trace operation is over the 3 principal dipole orientations.

There is one potential problem with Van Veen and colleagues' approach for the neuronal activity index using equation 10: For some dipole locations, if one principal orientation generates a much weaker lead-field than the other two principal orientations do, both the numerator and denominator of equation 10 will be dominated by the unreliable weak dipole orientation and not by the other stronger orientations as they should be. One example of this is a realistic forward head model such as a boundary element model (BEM) in MEG data analysis. Unlike the spherical MEG head model, where radial dipole orientation generates zero magnetic field, the realistic MEG head model using the BEM will generate a non-zero, but much weaker MEG lead-field compared with the two tangential orientations. As a consequence, equation 10 will be dominated by this unreliable radial dipole moment. This problem is due to first adding the total power among three orientations and then dividing the sum by the total noise power among all three orientations. We propose an alternative method: to divide the individual source power of each direction by its corresponding noise power first, and then add the ratios together later:

$$
\begin{gathered}
R_{\text {type_1_alt }}=\frac{\left(\mathbf{1}_{x}^{T} \mathbf{C}^{-1} \mathbf{1}_{x}\right)_{i}^{-1}}{\left(\mathbf{1}_{x}^{T} \Sigma^{-1} \mathbf{1}_{x}\right)_{i}^{-1}}+\frac{\left(\mathbf{1}_{y}^{T} \mathbf{C}^{-1} \mathbf{1}_{y}\right)_{i}^{-1}}{\left(\mathbf{1}_{y}^{T} \Sigma^{-1} \mathbf{1}_{y}\right)_{i}^{-1}}+\frac{\left(\mathbf{1}_{z}^{T} \mathbf{C}^{-1} \mathbf{1}_{z}\right)_{i}^{-1}}{\left(\mathbf{1}_{z}^{T} \Sigma^{-1} \mathbf{1}_{z}\right)_{i}^{-1}} \\
\text { for } i=1,2, \ldots, P
\end{gathered}
$$

Now, the problem of a weak orientation overshadowing the other stronger orientations no longer exists. 


\section{Vectorized Type 2 Beamformer}

Sekihara and colleagues (2001) reported a beamformer approach in MEG, which purportedly was based on work originally introduced by Borgiotti and Kaplan (1979). The authors also stated that this beamformer was different from the well-known minimum-variance beamformer. However, our examination of Borgiotti and Kaplan's original paper in 1979 showed that their beamformer is identical to the linearly-constrained minimum-variance beamformer described by equation 4 through equation 9 . The only deviation was that they used a different measure from the one used by Van Veen and colleagues (equation 10) to describe the spatial distribution of the activity of the sources. Here, we present our derivation on the vectorized Borgiotti-Kaplan's beamformer for the EEG/MEG neuronal activity index. We will then compare our version of Borgiotti-Kaplan's beamformer with that of Sekihara and colleagues.

In Borgiotti and Kaplan (1979), $\left(\mathbf{w}^{\mathrm{T}} \mathbf{C} \mathbf{w}\right) /\left(\mathbf{w}^{\mathrm{T}} \mathbf{w}\right)$ was used to describe the source activity where the beamformer weights $\mathbf{w}$ took the identical form to the minimum-variance beamformer shown previously in equation 8. Following the same approach, we obtained the vectorized version of the Borgiotti-Kaplan's beamformer (type 2) to express the neuronal activity index for the entire interval that was used to evaluate the covariance matrix $\mathbf{C}$ :

$$
\begin{gathered}
R_{\text {type } \_2}=\frac{\left(\mathbf{1}_{x}^{T} \mathbf{C}^{-1} \mathbf{1}_{x}\right)_{i}}{\left(\mathbf{1}_{x}^{T} \mathbf{C}^{-2} \mathbf{1}_{x}\right)_{i}}+\frac{\left(\mathbf{1}_{y}^{T} \mathbf{C}^{-1} \mathbf{1}_{y}\right)_{i}}{\left(\mathbf{1}_{y}^{T} \mathbf{C}^{-2} \mathbf{1}_{y}\right)_{i}}+\frac{\left(\mathbf{1}_{z}^{T} \mathbf{C}^{-1} \mathbf{1}_{z}\right)_{i}}{\left(\mathbf{1}_{z}^{T} \mathbf{C}^{-2} \mathbf{1}_{z}\right)_{i}} \\
\text { for } i=1,2, \ldots, P
\end{gathered}
$$

Since the lead-field is in both the numerator and denominator in the right side of equation 13 , one does not have to worry about the erroneously large values near the center of the head. The dipole time-courses can be obtained from equation 4 and equation 8 , just like the standard minimum-variance beamformer. The neuronal activity index of this type 2 beamformer for each time point can be expressed as:

$$
\begin{aligned}
r_{\text {type } 2}(t)= & \frac{\left(\mathbf{w}_{x}^{T} \mathbf{b}(t) \mathbf{b}(t)^{T} \mathbf{w}_{x}\right)_{i}}{\left(\mathbf{w}_{x}^{T} \mathbf{w}_{x}\right)_{i}}+\frac{\left(\mathbf{w}_{y}^{T} \mathbf{b}(t) \mathbf{b}(t)^{T} \mathbf{w}_{y}\right)_{i}}{\left(\mathbf{w}_{y}^{T} \mathbf{w}_{y}\right)_{i}}+ \\
& \frac{\left(\mathbf{w}_{z}^{T} \mathbf{b}(t) \mathbf{b}(t)^{T} \mathbf{w}_{z}\right)_{i}}{\left(\mathbf{w}_{z}^{T} \mathbf{w}_{z}\right)_{i}} \text { for } i=1,2, \ldots, P
\end{aligned}
$$

To be more explicit, the first term in the right-hand side of equation 14a can be written as:

$$
\frac{\left(\mathbf{w}_{x}^{T} \mathbf{b}(t) \mathbf{b}(t)^{T} \mathbf{w}_{x}\right)_{i}}{\left(\mathbf{w}_{x}^{T} \mathbf{w}_{x}\right)_{i}}=\frac{\left(\mathbf{1}_{x}^{T} \mathbf{C}^{-1} \mathbf{b}(t) \mathbf{b}(t)^{T} \mathbf{C}^{-1} \mathbf{1}_{x}\right)_{i}}{\left(\mathbf{1}_{x}^{T} \mathbf{C}^{-2} \mathbf{1}_{x}\right)_{i}}
$$

Sekihara and colleagues (2001) stated that they used the beamformer approach from Borgiotti and Kaplan in 1979. However, we found two major differences between Sekihara et al. (2001) and Borgiotti and Kaplan (1979). The first difference was that Borgiotti and Kaplan used exactly the same linearly-constrained minimum-variance beamformer by assuming $\mathbf{w}^{\mathrm{T}} \mathbf{l}$ to be a constant (usually set to 1 see equation 7) for all the designated beams. In Sekihara et al. (2001), $\mathbf{w}^{\mathrm{T}} \mathbf{1}$ was originally claimed to be a constant, but later it was set to be a complicated function of the lead-fields and the signal covariance matrix. As a consequence, the beamformer weight $\mathbf{w}$ in Sekihara et al. (2001) took a different format from that of the standard minimum-variance beamformer. The second difference was that Borgiotti and Kaplan (1979) used $\left(\mathbf{w}^{\mathrm{T}} \mathbf{C} \mathbf{w}\right) /\left(\mathbf{w}^{\mathrm{T}} \mathbf{w}\right)$ to describe the source activity for the entire interval. The corresponding source activity for any time point would be $\left[\mathbf{w}^{\mathrm{T}} \mathbf{b}(t) \mathbf{b}(t)^{\mathrm{T}} \mathbf{w}\right] /\left(\mathbf{w}^{\mathrm{T}} \mathbf{w}\right)$, as shown in equation 14. Sekihara et al. (2001) did not provide a formula of the neuronal source activity for the entire interval. Instead they used $\mathbf{w}^{\mathrm{T}} \mathbf{b}(t) \mathbf{b}(t)^{\mathrm{T}} \mathbf{w}$ for the source activity for any time point using their expression of the beamformer coefficients.

A very interesting point is that the second difference between Sekihara et al. (2001) and Borgiotti and Kaplan (1979) cancelled the first difference in their approaches. As a consequence, if one follows the steps of Sekihara et al. (2001), one would get the same equation for the neuronal activity index at each time point as we did in equation 14 for the Borgiotti and Kaplan's beamformer. Therefore, while the simulations presented in Sekihara et al. (2001) still hold, it is difficult to follow their derivation. Moreover, since their beamformer coefficients took a different format from the minimum-variance beamformer, Sekihara and colleagues claimed that Borgiotti and Kaplan's beamformer was different from the well-known minimum-variance beamformer. However, our derivation shown earlier demonstrates that Borgiotti and Kaplan's beamformer is exactly the same as the minimum-variance beamformer in terms of beamformer coefficients. Borgiotti and Kaplan just used a different expression for the source spatial activity index than the other minimum-variance beamformer approaches (e.g., type 1 beamformer by Van Veen's and colleagues).

Another topic discussed by Sekihara et al. (2001) was the use of signal-space projection to clean up the dipole time-courses. In this approach, eigenvalue decomposition was performed on the data covariance matrix $\mathbf{C}$, and the entire eigenspace was truncated into signal subspace 
(with large eigenvalues) and noise subspace (with small eigen values). Since $\mathbf{C}$ is a square matrix, a singular-value decomposition of $\mathbf{C}$ will be equivalent to the eigenvalue decomposition (the singular vectors will be the same as the eigenvectors, and the singular values will be the square root of the eigenvalues). Then, the beamformer coefficient $\mathbf{w}$ was projected into the signal subspace:

$$
\overline{\mathbf{w}}_{k}=\mathbf{E}_{s} \mathbf{E}_{s}^{T} \mathbf{w}_{k} \quad \text { for } k=1,2, \ldots, 3 P
$$

where the columns of $\mathbf{E}_{s}$ contains the eigenvectors in the signal subspace. This step can be considered as a truncated regularization approach using subspace projection (Mosher et al. 1993).

\section{Vectorized Type 3 Beamformer}

Another well-known beamformer was presented by Robinson and Vrba (1999). It was also known as synthetic aperture magnetometry (SAM). Since SAM was clearly described as a minimum-variance beamformer (Robinson and Vrba 1999), many researchers (e.g. Sekihara et al. 2001; Barnes and Hillebrand 2003) refer to it as the same minimum beamformer as the one from Van Veen and colleagues (1997). However, our analysis will show substantial differences between these two beamformer approaches.

First of all, the beamformer from Robinson and Vrba is indeed a linearly-constrained minimum-variance beamformer. Therefore, the expressions for the vectorized version of this beamformer are identical to equation 5 through equation 8 . However, Robinson and Vrba used a different way to describe the neuronal activity at the ith dipole location for the entire time interval in which the covariance matrix was estimated. A vectorized version of their beamformer approach (type 3) is:

$$
\begin{aligned}
R_{\text {type } 3}= & \frac{\left(\mathbf{w}_{x}^{T} \mathbf{C} \mathbf{w}_{x}\right)_{i}}{\left(\mathbf{w}_{x}^{T} \Sigma \mathbf{w}_{x}\right)_{i}}+\frac{\left(\mathbf{w}_{y}^{T} \mathbf{C} \mathbf{w}_{y}\right)_{i}}{\left(\mathbf{w}_{y}^{T} \Sigma \mathbf{w}_{y}\right)_{i}}+\frac{\left(\mathbf{w}_{z}^{T} \mathbf{C} \mathbf{w}_{z}\right)_{i}}{\left(\mathbf{w}_{z}^{T} \Sigma \mathbf{w}_{z}\right)_{i}} \\
& =\frac{\left(\mathbf{1}_{x}^{T} \mathbf{C}^{-1} \mathbf{1}_{x}\right)_{i}}{\left(\mathbf{1}_{x}^{T} \mathbf{C}^{-1} \Sigma \mathbf{C}^{-1} \mathbf{1}_{x}\right)_{i}}+\frac{\left(\mathbf{1}_{y}^{T} \mathbf{C}^{-1} \mathbf{1}_{y}\right)_{i}}{\left(\mathbf{1}_{y}^{T} \mathbf{C}^{-1} \Sigma \mathbf{C}^{-1} \mathbf{1}_{y}\right)_{i}} \\
& +\frac{\left(\mathbf{1}_{z}^{T} \mathbf{C}^{-1} \mathbf{1}_{z}\right)_{i}}{\left(\mathbf{1}_{z}^{T} \mathbf{C}^{-1} \Sigma \mathbf{C}^{-1} \mathbf{1}_{z}\right)_{i}} \quad \text { for } i=1,2, \ldots, P
\end{aligned}
$$

Thus, it is clear that this type 3 beamformer (equation 16) based on Robinson and Vrba's approach is substantially different from the type 1 beamformer from Van
Veen and colleagues (equation 10). However, the type 3 beamformer is quite similar to the vectorized type 2 beamformer from Borgiotti and Kaplan (equation 13). If the noise in the data is pure white and identically distributed across sensors, the data covariance matrix $\sum$ will be an identity matrix times a constant. In this case, there will be no difference between the un-regularized type 3 beamformer and the un-regularized version of the type 2 beamformer.

The dipole time-courses of the type 3 beamformer can be obtained from equation 4 and equation 8 , just like the standard minimum-variance beamformer. We can also obtain the vectorized neuronal activity index for dipole $i$ at each time point:

$$
\begin{aligned}
r_{\text {type } 3}(t) & =\frac{\left(\mathbf{w}_{x}^{T} \mathbf{b}(t) \mathbf{b}(t)^{T} \mathbf{w}_{x}\right)_{i}}{\left(\mathbf{w}_{x}^{T} \Sigma \mathbf{w}_{x}\right)_{i}}+\frac{\left(\mathbf{w}_{y}^{T} \mathbf{b}(t) \mathbf{b}(t)^{T} \mathbf{w}_{y}\right)_{i}}{\left(\mathbf{w}_{y}^{T} \Sigma \mathbf{w}_{y}\right)_{i}} \\
& +\frac{\left(\mathbf{w}_{z}^{T} \mathbf{b}(t) \mathbf{b}(t)^{T} \mathbf{w}_{z}\right)_{i}}{\left(\mathbf{w}_{z}^{T} \Sigma \mathbf{w}_{z}\right)_{i}} \quad \text { for } i=1,2, \ldots, P
\end{aligned}
$$

To be more explicit, the first term of the right-hand side of equation 17a can be expressed as:

$$
\frac{\left(\mathbf{w}_{x}^{T} \mathbf{b}(t) \mathbf{b}(t)^{T} \mathbf{w}_{x}\right)_{i}}{\left(\mathbf{w}_{x}^{T} \Sigma \mathbf{w}_{x}\right)_{i}}=\frac{\left(\mathbf{1}_{x}^{\mathrm{T}} \mathbf{C}^{-1} \mathbf{b}(t) \mathbf{b}(t)^{T} \mathbf{C}^{-1} \mathbf{1}_{x}\right)_{i}}{\left(\mathbf{1}_{x}^{T} \mathbf{C}^{-1} \Sigma \mathbf{C}^{-1} \mathbf{1}_{x}\right)_{i}}
$$

To handle the case of noisy data, Robinson and Vrba (1999) also used a Backus-Gilbert regularization, in which the signal covariance matrix $\mathbf{C}$ in equation 16 was replaced by $C+\mu \Sigma$, where $\mu$ is the regularization factor.

\section{A Novel Vectorized Beamformer using Higher- Order Covariance - Type 4}

By now, it should be clear that all existing beamformers for EEG and MEG analysis are linearly-constrained minimum-variance beamformers, and they are identical up to the point of deriving the beamformer coefficients $\mathbf{w}_{k}$. The beamformers differ in terms of their different expressions for the neuronal activity index. We now introduce a novel way to express the neuronal activity index using a higher-order covariance matrix. In our approach (type 4 beamformer), the covariance matrix used in the minimum-variance beamformer coefficients (equation 8) is replaced by its higher-order expression. So, the form of the neuronal activity index for the $i$ th dipole for the entire interval where the covariance matrix was estimated can be expressed as: 


$$
\begin{gathered}
R_{\text {type } 4}=\frac{\left(\mathbf{1}_{x}^{T} \mathbf{C}^{-n} \mathbf{1}_{x}\right)_{i}^{-1}}{\left(\mathbf{1}_{x}^{T} \Sigma^{-n} \mathbf{1}_{x}\right)_{i}^{-1}}+\frac{\left(\mathbf{1}_{y}^{T} \mathbf{C}^{-n} \mathbf{1}_{y}\right)_{i}^{-1}}{\left(\mathbf{1}_{y}^{T} \Sigma^{-n} \mathbf{1}_{y}\right)_{i}^{-1}}+\frac{\left(\mathbf{1}_{z}^{T} \mathbf{C}^{-n} \mathbf{1}_{z}\right)_{i}^{-1}}{\left(\mathbf{1}_{z}^{T} \Sigma^{-n} \mathbf{1}_{z}\right)_{i}^{-1}} \\
\text { for } i=1,2, \ldots, P
\end{gathered}
$$

where $n>1$ is the order of the data covariance matrix. This expression is similar to our modified version of the type 1 beamformer of Van Veen and colleagues presented earlier (equation 12), but with a higher-order covariance matrix. For our beamformer the dipole time-course was obtained from equation 4 and equation 8 by replacing the covariance matrices $\mathrm{C}$ and $\Sigma$ with $\mathbf{C}^{n}$ and $\Sigma^{n}$, respectively. The neuronal activity index for the ith dipole at any time point can be expressed as:

$$
\begin{aligned}
r_{\text {type_4 }}(t) & =\frac{\left[\mathbf{w}_{x}^{T}\left[\mathbf{b}(t) \mathbf{b}(t)^{T}\right]^{n} \mathbf{w}_{x}\right]_{i}}{\left(\mathbf{1}_{x}^{T} \Sigma^{-n} \mathbf{1}_{x}\right)_{i}^{-1}}+\frac{\left\lfloor\mathbf{w}_{y}^{T}\left[\mathbf{b}(t) \mathbf{b}(t)^{T}\right]^{n} \mathbf{w}_{y}\right\rfloor_{i}}{\left(\mathbf{1}_{y}^{T} \Sigma^{-n} \mathbf{1}_{y}\right)_{i}^{-1}} \\
& +\frac{\left[\mathbf{w}_{z}^{T}\left[\mathbf{b}(t) \mathbf{b}(t)^{T}\right]^{n} \mathbf{w}_{z}\right]_{i}}{\left(\mathbf{1}_{z}^{T} \Sigma^{-n} \mathbf{1}_{z}\right)_{i}^{-1}} \text { for } i=1,2, \ldots, P
\end{aligned}
$$

where the beamformer coefficients $\mathbf{w}$ were obtained using equation 8 but replacing $\mathbf{C}$ with $\mathbf{C}^{n}$. The advantage of our beamformer in comparison to the other approaches is illustrated in the Results section.

\section{Simulation Setup}

First, we examined the performance of different beamformers using simulated MEG data. In the simulation, we adopted a two-dimensional dipole grid as the imaging space inside a spherical head. The dipole grid contains 51 columns and 26 rows with an equal mesh size of $2 \mathrm{~mm}$. A Neuromag-122 (Neuromag, Helsinki, Finland) whole-head system with 122 channels of planar-gradiometers was used in the simulation. The relative location of the image space with respect to the Neuromag-122 sensors is shown in figure 1 . The $x$-axis in the figure denotes the left-right direction with positive $x$ to the right, the $y$-axis denotes the anterior-posterior direction with positive $y$ to the anterior direction, and the $z$-axis was in the superior-inferior direction with positive $z$ to the superior direction.

Lead-fields were calculated using the Sarvas (1985) formula. Since the radial dipole orientation does not generate magnetic fields for the spherical head model, the principal orientations used in the beamformer analysis are reduced to two, namely in the directions of the eleva- tion angle $\theta$ and the azimuth angle $\boldsymbol{\phi}$. Two closely-spaced dipoles at $[-0.8,0,7.8] \mathrm{cm}$ and $[0,0,8] \mathrm{cm}$, respectively, were used to generate simulated MEG signals. The simulated dipole orientations for both dipoles were in the positive y direction. During the first simulation, the two dipoles were assigned nearly uncorrelated time-courses. Different SNR levels were examined by adding white noise to the simulated MEG response. The SNR in the present study was defined as the ratio of the root-mean-square (RMS) value of the total MEG signal to that of the white noise for all 122 sensors within given time intervals. This SNR definition was similar to the one defined by the ratio of Frobenius norms used by Sekihara et al. (2001), and it is also the same as the square-root of the SNR used by Van Veen et al. (1997), which was defined as the ratio of the signal variances to noise variance.

The four vectorized beamformers described in the Method section were studied in the simulation: 1) type 1 beamformer based on the work of Van Veen and colleagues (equation 10); (2) type 2 beamformer based on the work of Borgiotti and Kaplan (equation 13) with regularization using signal-space projection (equation 15) by Sekihara and colleagues; (3) type 3 beamformer based on the work on Robinson and Vrba (equation 16) with Backus-Gilbert regularization; and (4) Our type 4 beamformer (equation 18), using the higher-order covariance matrix. The performance of these beamformers was measured by their ability to (1) effectively resolve two closely-spaced dipoles with parallel orientations and (2) suppress false-positive activities shown as "activities" not at the locations of the true sources.

\section{Human Median-Nerve MEG Response}

In order to investigate the real-world performance of these beamformers, we also applied the 4 different beamformers to an MEG data set from a unilateral median-nerve stimulation paradigm. The data were collected from a healthy male volunteer, age 37 , with no history of head injury or neurological disorder. The consent form was approved by the Human Research Review Committee at the University of New Mexico.

During the median-nerve test, the right median-nerve of the subject was stimulated using a bipolar Grass ${ }^{\circledR}$ constant current stimulator. The stimuli were square-wave electric pulses ( $0.2 \mathrm{~ms}$ duration) delivered at $1 \mathrm{~Hz}$. The intensity of the stimulation was adjusted until robust thumb twitches were observed. A trigger from the stimulator, which was simultaneous with the stimulus, was sent to the MEG acquisition system for signal averaging. Magnetic fields evoked by median-nerve stimulation were measured using a Neuromag ${ }^{\circledR}$ whole-head MEG system (Helsinki, Finland), with 122 planar gradiometers in a magnetically shielded room 


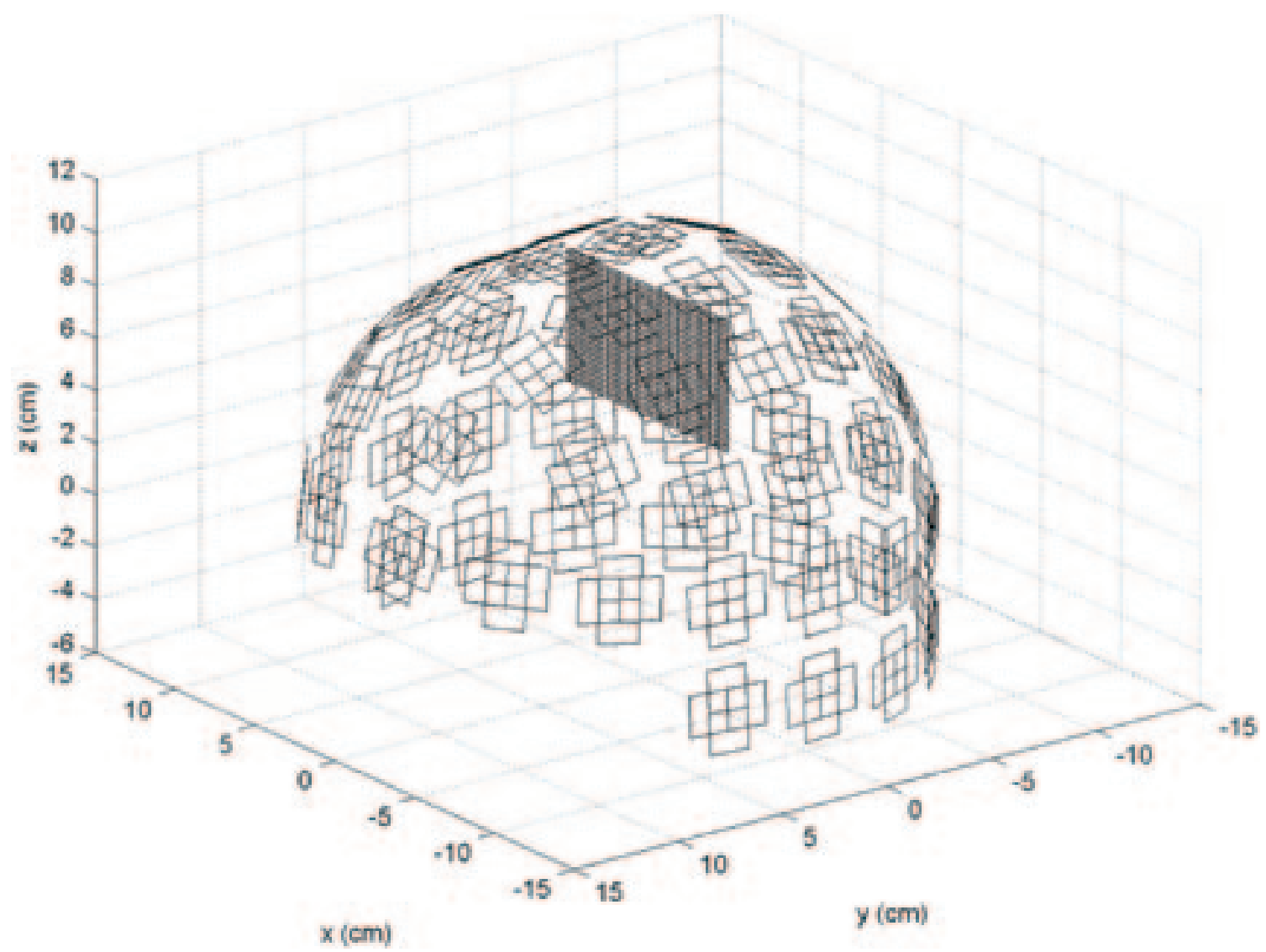

Figure 1. Dipole grid and Neuromag-122 MEG sensory array used in our simulations. The dipole grid contains 51 columns and 26 rows with an equal mesh size of $2 \mathrm{~mm}$. The whole-head MEG system contains 122 channels of planar-gradiometers.

(IMEDCO-AG, Switzerland). EOG electrodes were used to detect eye blinks and eye movements. An interval of $500 \mathrm{~ms}$ post-stimulus was recorded, using $300 \mathrm{~ms}$ pre-stimulus data for noise estimation. Data were sampled at $1000 \mathrm{~Hz}$ and run through a high-pass filter with $0.1 \mathrm{~Hz}$ cut-off and through a notch filter $(58-62 \mathrm{~Hz})$ to remove $60 \mathrm{~Hz}$ power-line noise. Eight hundred artifact-free MEG responses were averaged with respect to the stimulus trigger to increase the SNR.

\section{Results}

\section{Signals with Very Low Correlation}

Two nearly un-correlated dipole time-courses (shown in figures $2 a$ and $2 b$ ) were used in our first simulation. The correlation between these two time-courses was $2.06 \%$. The dipole time-courses contained $300 \mathrm{~ms}$ of pre-stimulus and $400 \mathrm{~ms}$ of post-stimulus intervals. Noiseless MEG fields were calculated using these dipole time-courses. Then white noise was added to the data. Figures $3 \mathrm{a}$ and $3 \mathrm{~b}$ show the noisy MEG sensor waveforms with all 122 channels superimposed for a SNR $=6$ and 2, respectively. The signal covariance matrix $\mathbf{C}$ was estimated using the post-stimulus interval, while the noise covariance matrix $\Sigma$ was estimated using the pre-stimulus interval.

Figure 4 shows the neuronal activity index maps for $\mathrm{SNR}=6$ using the four beamformers. In each subplot, the scale of the neuronal activity index was normalized to its maximum value. It is clear that at this SNR level all four beamformers can separate these two closely-spaced parallel dipoles, although the separation of the two dipoles using the type 1 beamformer was not as good as the other three. The non-zero baseline shifts in the type 2 (figure $4 b$ ) and type 3 (figure $4 \mathrm{c}$ ) beamformers were mainly the result of the regularization. The dimension of the signal subspace used in the regularization of the type 2 beamformer was 2. The Backus-Gilbert regularization parameter $\mu$ used in the type 3 beamformer was determined by the method proposed by Gross and Ioannides (1999). A third-order covariance matrix $(n=3)$ was used in our type 4 beamformer (figure $4 \mathrm{~d}$ ). 
(a) Dipole 1: uncorrelated case

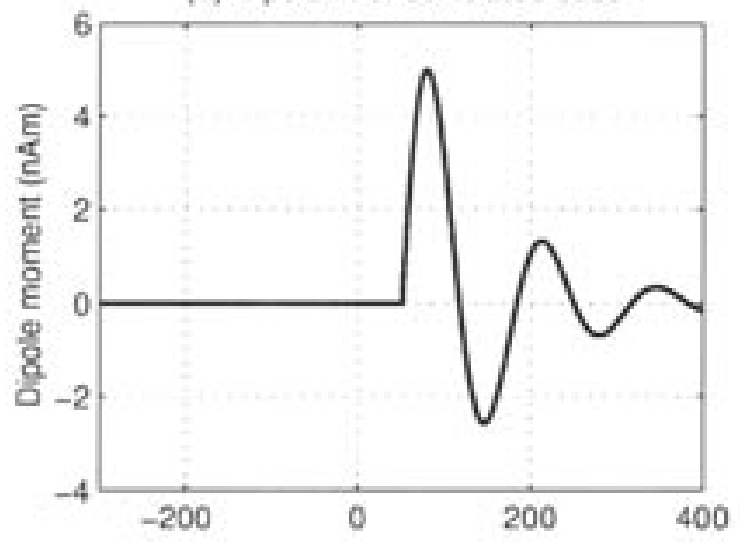

(b) Dipole 2: uncorrelated case

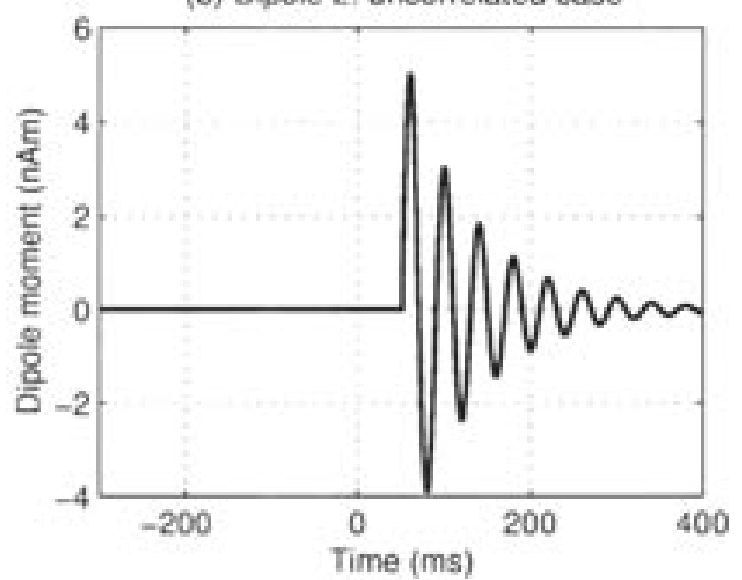

(c) Dipole 1: correlated case

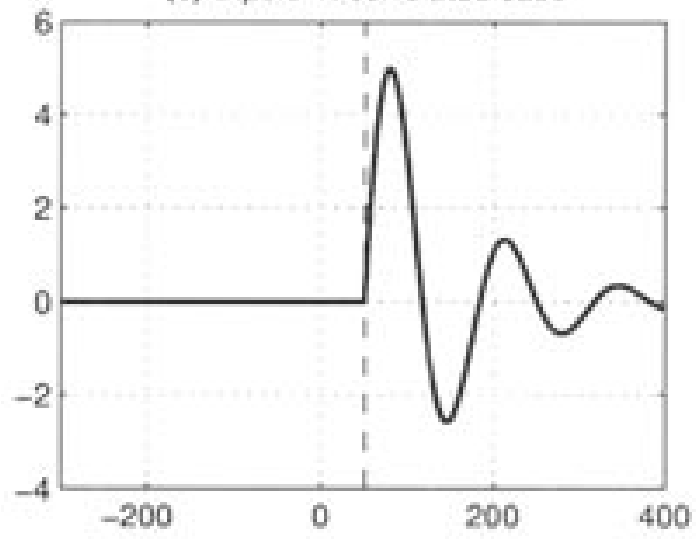

(d) Dipole 2: correlated case

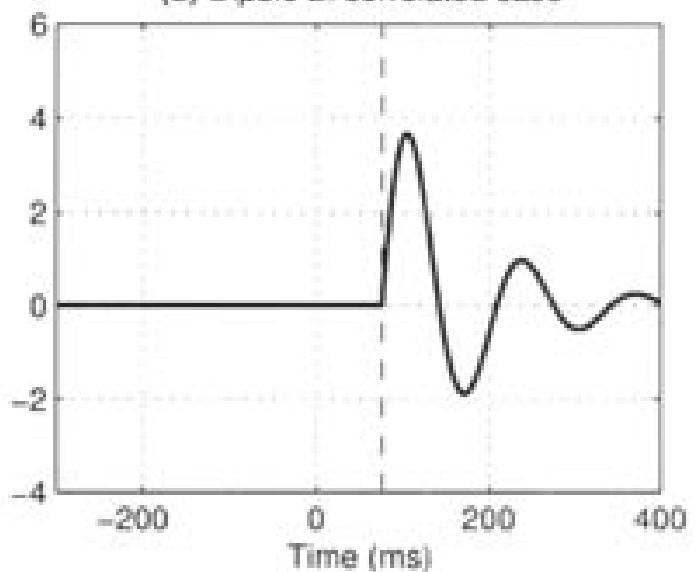

Figure 2. Dipole time-courses used in the simulations. (a)(b): two nearly un-correlated dipole time-courses (2.06\% correlation) used in the un-correlated simulation. (c)(d): two correlated dipole time-courses used in the correlated simulation, in which the time-course of Dipole 1 in (c) was kept to be the same as in (a) and the time-course of Dipole 2 in (d) was taken to be the same shape as Dipole 1 but with time delays of 20 or $10 \mathrm{~ms}$.

Figure 5 shows that greater differences were observed among the beamformers in the case of $\mathrm{SNR}=2$. The type 1 beamformer (figure 5a) was unable to separate the two closely-spaced parallel dipoles and only one peak was observed in the neuronal activity index map. The background of the type 1 beamformer was quite clean, though, suggesting low false-positive activity. One the other hand, the type 2 beamformer (subspace dimension of 2 ) in figure $5 b$ showed two major peaks corresponding to the two dipoles. However, several additional smaller peaks were also present in the reconstruction, indicating possible false-positives. Similarly, the type 3 beamformer showed two major peaks with additional smaller local maxima (figure 5c). These results contrast with our type 4 beamformer $(n=3)$, in which the two closely-spaced parallel sources were clearly distinguishable and the background was also clean with no visible false-positive activity (figure $5 d$ ).

\section{Correlated Signals}

In this simulation, performance of the 4 different beamformers was studied using correlated dipole time-courses. The time-course of dipole 1 was kept to be the same as in the un-correlated case (figure 2c), and the time-course of dipole 2 was created to be the same shape as dipole 1, but with $20 \mathrm{~ms}$ and $10 \mathrm{~ms}$ time delays (figure $2 \mathrm{~d})$. The time-courses of the signal from the two dipole sources correlated $61 \%$ and $90 \%$, respectively. White noise was added to the simulated MEG fields with $\mathrm{SNR}=6$ for both time-delays. The MEG sensor waveforms are shown in figure 6 with 122 channels superimposed.

Figure 7a shows the normalized neuronal activity index map using the type 1 beamformer for the lower-correlation case $(61 \%)$, which was able to separate the two closely-spaced dipoles, but the separation was not very good. Better separation was obtained from the type 2 
(a) $\mathrm{SNR}=6$

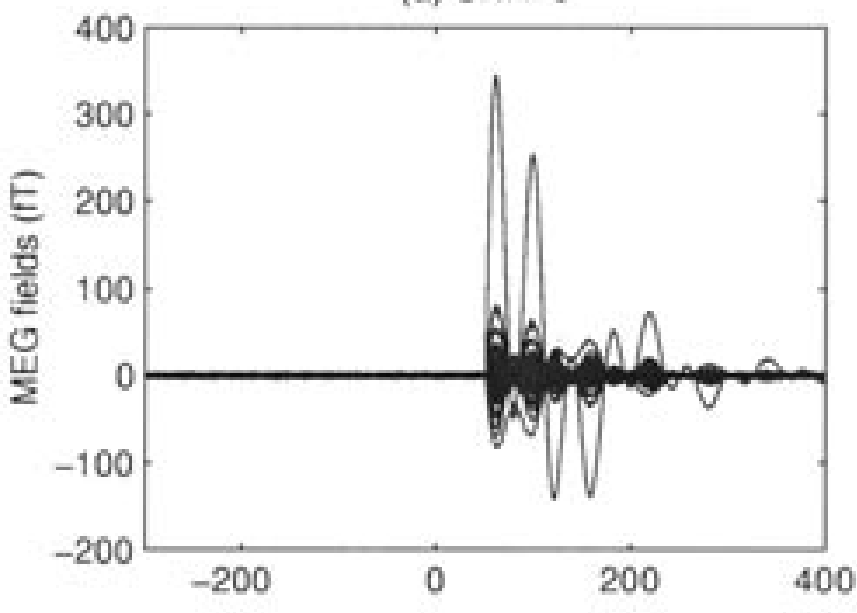

(b) $\mathrm{SNR}=2$

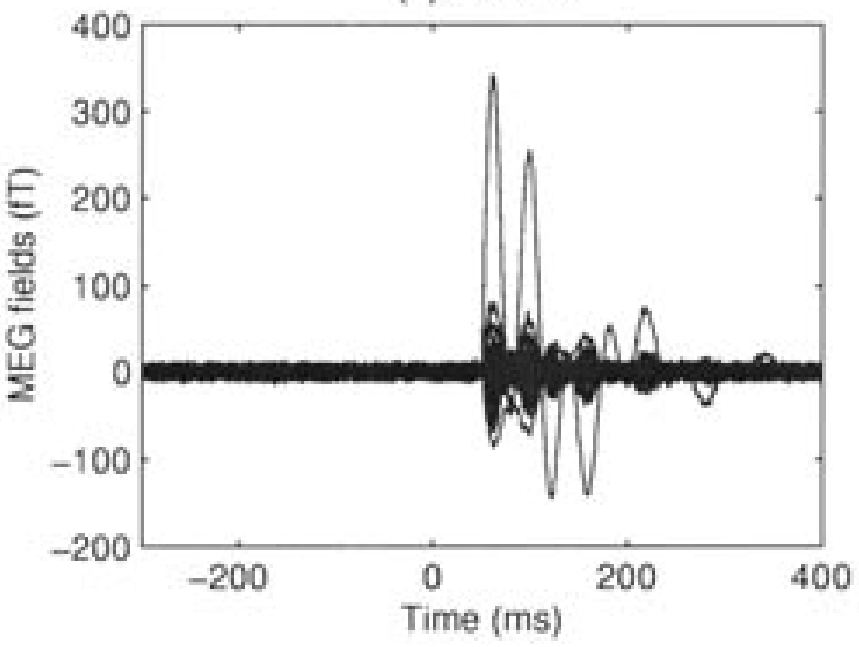

Figure 3. Simulated MEG fields for the nearly un-correlated simulation with different SNRs. The signals from 122 channels were superimposed. (a) $S N R=6$, (b) $S N R=2$.

beamformer (signal subspace dimension of 2), but additional smaller peaks were visible even at some distance from the real sources (figure $7 \mathrm{~b}$ ). Similarly, the type 3 beamformer showed two major peaks and some additional smaller peaks both close to and far from the real sources (figure 7c). On the other hand, our type 4 beamformer $(n=3)$ clearly separated these two closely-spaced parallel dipoles and had a clean background (figure 7d).

For the more challenging case of $90 \%$ correlation between the time-courses of these two dipoles, the type 1 beamformer (figure 8a) was no longer able to separate the two-closely spaced dipoles; there was however no visible false-positive activity in the background. In contrast, the type 2 beamformer (figure $8 \mathrm{~b}$, signal subspace dimension of two) separated the two dipoles, but there were additional relatively large peaks, indicating that the background was not very clean. Similar results were seen using the type 3 beamformer (figure 8c). On the other hand, our type 4 beamformer $(n=3)$ distinguished the two highly-correlated dipoles and the background remained clean, with no visible false-positive activity (figure 8d).

\section{Source Orientations and the Order of Covariance Matrix}

Vectorized beamformer approaches (equations 10, 13,16 , and 18) are thought to work for any dipole orientation. The choice of dipole along one of the orthogonal axes is purely for convenience, as was done in other papers (Van Veen et al. 1997; Gross and Ioannides 1999; Sekihara et al. 2001; Barnes and Hillebrand 2003). To confirm this principle, we rotated the dipole orientations in the simulation 45 degrees along the $\mathrm{z}$-axis, and obtained results that were virtually identical to the findings before the rotation, for all simulated cases shown previously in this section.

We also tested the performances of our type 4 beamformer for different orders of the covariance matrix for all simulated cases. Among all different orders, the third-order covariance matrix $(n=3)$ provided the best spatial resolution. For $n=4$ or higher, saturation was observed in the neuronal activity index map, whereas for $n=2$, the spatial resolution was between the results of $n=3$ and the type 1 beamformer of Van Veen et al. (1997).

\section{Results for Human Median-Nerve MEG Response}

Figure 9 shows the averaged MEG sensor waveforms (122 channels superimposed) evoked by right median-nerve stimulation of a normal human subject. The spike around $0 \mathrm{~ms}$ represents stimulus artifact. The signal covariance matrix was estimated using the 15 to 500 ms post-stimulus interval, and the noise-variance was estimated using the -300 to $-5 \mathrm{~ms}$ pre-stimulus interval. The SNR of the data set was estimated to be 4.55 . The image space used in the analysis was constructed using the cortical surface obtained from the subject's MRI and Neuromag ${ }^{\circledR}$ software. The top row of figure 10 shows the left, superior, and right views of the cortical surface used in this calculation. The entire image space contained 32,270 triangles and 16,137 dipole nodes with the side of each triangle about $2 \mathrm{~mm}$. The spherical head model (Sarvas 1995) was used to calculate the lead-fields for the dipole grid. Also shown in the top row of figure 10 are the expected sources based on existing neurophysiological knowledge of the median-nerve response (see Introduction for references). These sources are: primary somatosensory area for the wrist (SIw), pri- 
(a) Type 1 Beamformer

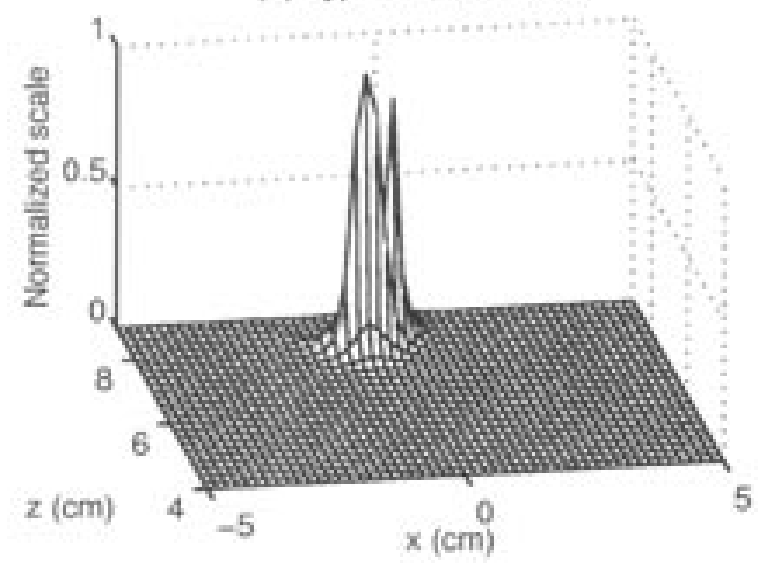

(c) Type 3 Beamformer

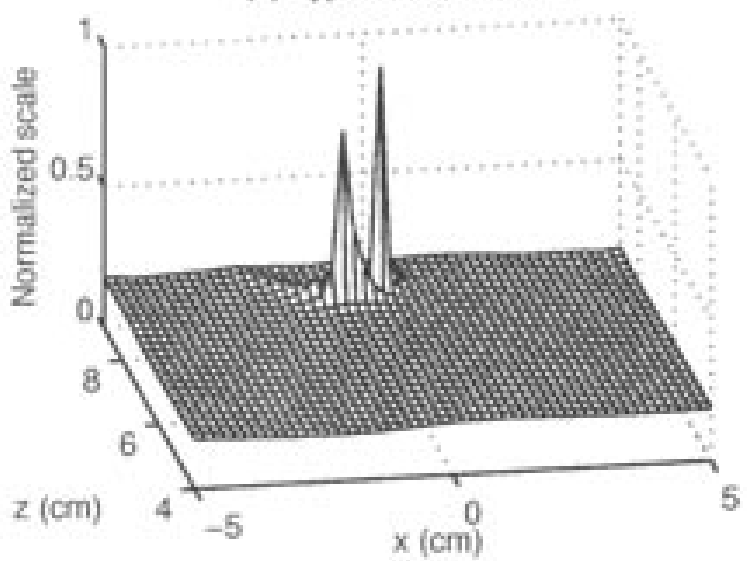

(b) Type 2 Beamtormer

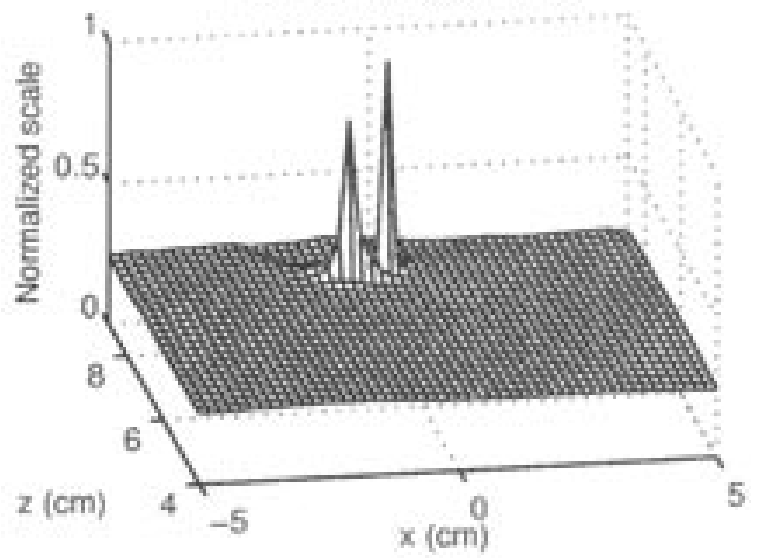

(d) Type 4 Beamformer

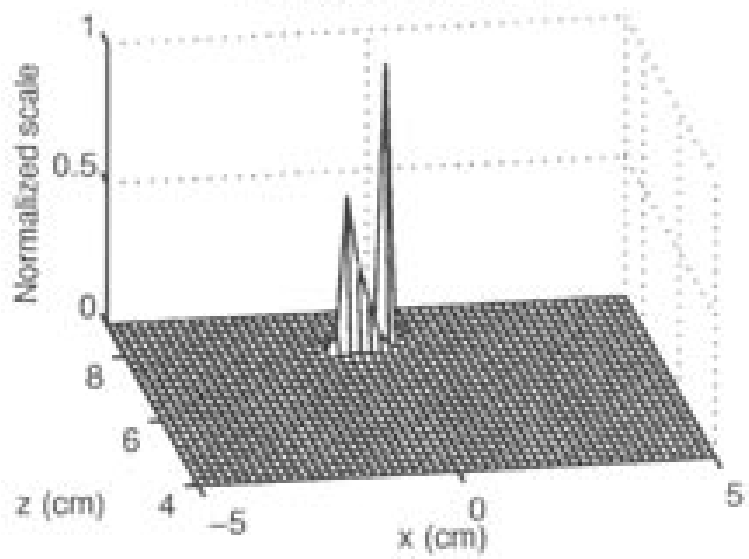

Figure 4. Normalized neuronal activity index for the two nearly un-correlated dipole sources using 4 different vectorized beamformers at SNR=6: (a) using type 1 beamformer based on work from Van Veen and colleagues, (b) using type 2 beamformer based on the work from Borgiotti and Kaplan with dimension of signal subspace of 2, (c) using type 3 beamformer based on the work from Robinson and Vrba with Backus-Gilbert regularization, and (d) using our type 4 beamformer with $3^{\text {rd }}$ order covariance.

mary somatosensory area for the thumb (SIt), primary motor area (MI), secondary somatosensory area (SII), premotor area (PrM), superior parietal area (Prtl), all contralateral (left hemisphere) to the side of the stimulation; plus the mid-line supplementary motor area (SMA) and the secondary somatosensory area ipsilateral (right hemisphere) to the stimulation (iSII).

In the remaining rows of figure 10, the neuronal activity index map for each beamformer approach is color-coded. In the color scale, 1.0 represents the maximum value of the index over all the 16,137 nodes, and 0.0 represents the minimum index value. The second row of figure 10 shows the neuronal activity index map obtained from the type 1 beamformer. An area in the contralateral (left) primary somatosensory area of the wrist, SIw, showed activation. However, in other ex- pected areas (i.e., SIt, MI, SII, PrM, SMA and iSII), activity was barely visible. Still, the background of the type 1 beamformer was very clean, as in the simulations.

The third row of figure 10 shows the results from the type 2 beamformer with a subspace dimension of 12 based on the singular-value decomposition of the covariance matrix. Unlike the type 1 beamformer, the type 2 beamformer showed considerable background activity in both contralateral and ipsilateral hemispheres. Contralateral SII and SIt showed strong activity. However, diffuse activity was seen in the SMA, and relatively weak activity appeared in MI, PrM, Prtl, and iSII. It was also difficult to distinguish the primary somatosensory area of the wrist SIw from the MI source (top-view).

The fourth row of figure 10 shows the results of from the type 3 beamformer. Again, considerable background 

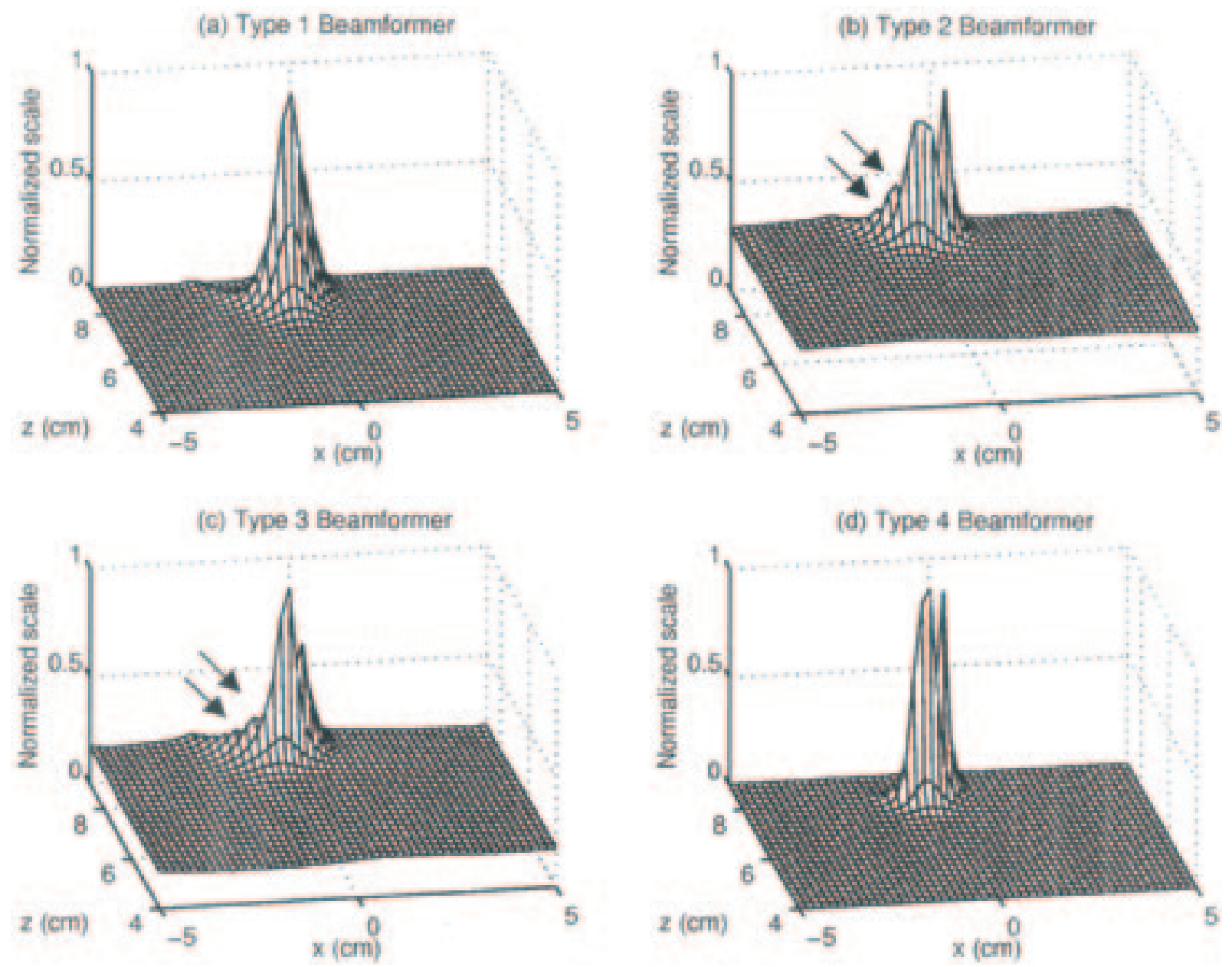

Figure 5. Normalized neuronal activity index for the two un-correlated dipole sources using 4 different vectorized beamformers at SNR=2: (a) The type 1 beamformer showed clean background but was unable to separate the two closely-spaced parallel dipoles. (b) The type 2 beamformer showed two major peaks corresponding to the two dipoles with additional smaller peaks (indicated by arrows) indicating possible false-positive activities. (c) The type 3 beamformer also showed two major peaks at the two dipole locations with additional smaller peaks. (d) Our type 4 beamformer distinguished the two dipoles with no visible additional false-positive activity.

activity was present. Strong activity was found in the SIt, SII, Prtl, and iSII. However, it was difficult to distinguish individual sources in one large area in the vicinity of the MI, PrM, and SMA. The SIw source was also missing in the map (top-view and right-view).

When our type 4 beamformer $(n=3)$ was applied to the same data set (bottom row of figure 10), strong and distinguishable activities were found in SIw, SIt, MI, SII, PrM, Prtl, and SMA, but weak activity was found in the iSII. Thus, almost all of the expected sources based on previous knowledge of human neurophysiology were reliably localized using our beamformer approach.

Another appealing feature of all the beamformer approaches is their low computational cost. For the large dipole grid used in our median-nerve MEG data analysis with 16,137 dipole sites, the computational time for any of the beamformers was less than 50 sec using MATLAB (Math Works Inc., Massachusetts, USA) on a $600 \mathrm{MHz}$ Pentium III PC. By comparison, a multiple dipole solution using nonlinear global optimization algorithms usually takes a couple of hours for this type of data (Huang et al. 1998; Uutela et al. 1998).

\section{Discussion}

\section{Performance of Beamformer Approaches}

In the present study, complete equations were provided for vectorized versions of existing beamformers in EEG and MEG source analysis. These equations included 
(a) $61 \%$ correlation

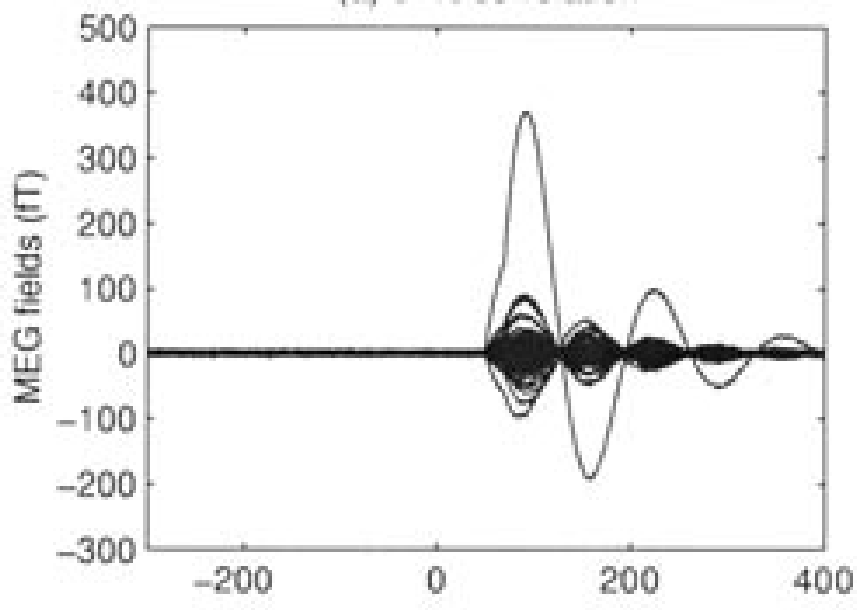

(b) $90 \%$ correlation

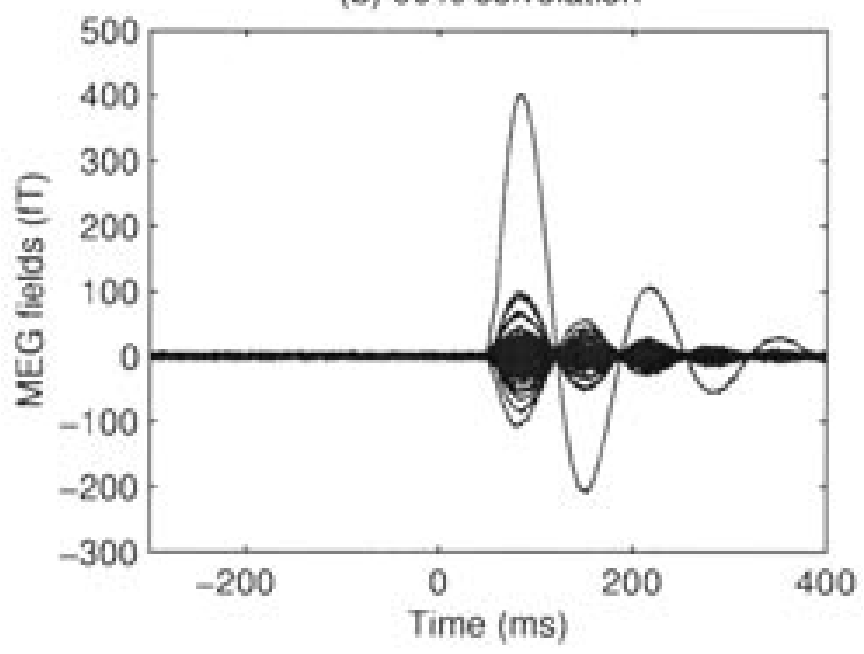

Figure 6. Simulated MEG fields for the correlated simulation with different levels of correlation between the two dipole sources and fixed $S N R=6$. The signals from 122 channels were superimposed: (a) correlation=61\%, (b) correlation $=90 \%$.

each beamformer's coefficients, source time-courses, and neuronal activity indices for both individual time points and the entire interval for which the data covariance matrix is calculated. These equations showed that all existing EEG and MEG beamformers are linearly-constrained minimum-variance beamformers and that they are identical in terms of the beamformer coefficients, if no regularization is used. Although Borgiotti-Kaplan's beamformer (type 2) was once thought to be different from the minimum-variance beamformer (Sekihara et al. 2001), our theoretical derivations based on the original framework from Borgiotti and Kaplan (1979) showed that this type of beamformer is clearly a minimum-variance beamformer.

Despite these commonalities, substantial differences sometimes existed theoretically and practically among the variety of existing EEG/MEG beamformers. These differences can all be characterized by the way in which the neuronal activity index is calculated. The beamformer from Van Veen et al. (1997, type 1) and another one from Robinson and Vrba (1999, type 3) have often been referred to as the same. However, the present analysis showed substantial differences not only in their theoretical expressions of the neuronal activity index, but also in their applications to simulated and empirical human data. At the same time, the difference between Borgiotti-Kaplan's beamformer using subspace regularization by Sekihara et al. (2001) and Robinson and Vrba's beamformer are not as substantial as previously claimed (Sekihara et al. 2001). The only differences between them are that Sekihara and colleagues used subspace projection while Robinson and Vrba used Backus-Gilbert regularization, and Robinson and Vrba used the noise covariance matrix while Sekihara and colleagues did not.

Computer simulation results using two closely-spaced dipoles, showed that for essentially uncorrelated dipole time-courses, the spatial resolution of the type 1 beamformer based on Van Veen and colleagues' approach decreased faster than that of both the type 2 beamformer based on Borgiotti and Kaplan's approach and the type 3 beamformer based on Robinson and Vrba's approach, when SNR decreased. A major advantage of the type 1 beamformer, however, was its ability to sustain clean background, whereas additional extra local peaks, not at the locations of the simulated dipoles, appeared in both the type 2 and type 3 beamformers at the low SNR. For correlated dipole time-courses, the spatial resolution of the type 1 beamformer decreased faster than those of the type 2 and type 3 beamformers as the correlation between two dipole time-courses increased. On the other hand, false-positive activity remained absent for the type 1 beamformer even at the high correlation level. This contrasted with both the type 2 and type 3 beamformers, which showed many additional local peaks, not at the locations of the simulated dipoles. Notably, the SNR of 6 and 2 used in the present simulations was much lower than the SNR used by other groups (e.g., SNR=18 used by Sekihara et al. 2001) and therefore, closer to the real situation in EEG and MEG data. At a SNR of 18, and for uncorrelated sources, the extra false local peaks that we observed at lower SNR levels become invisible (Sekihara et al. 2001).

We introduced a novel beamformer approach (type 4), which was based on higher-order covariance matrices. Computer simulations with un-correlated and correlated dipole sources showed that the type 4 beamformer's spatial resolution was better than the type 1 beamformer, and 
(a) Type 1 Beamformer

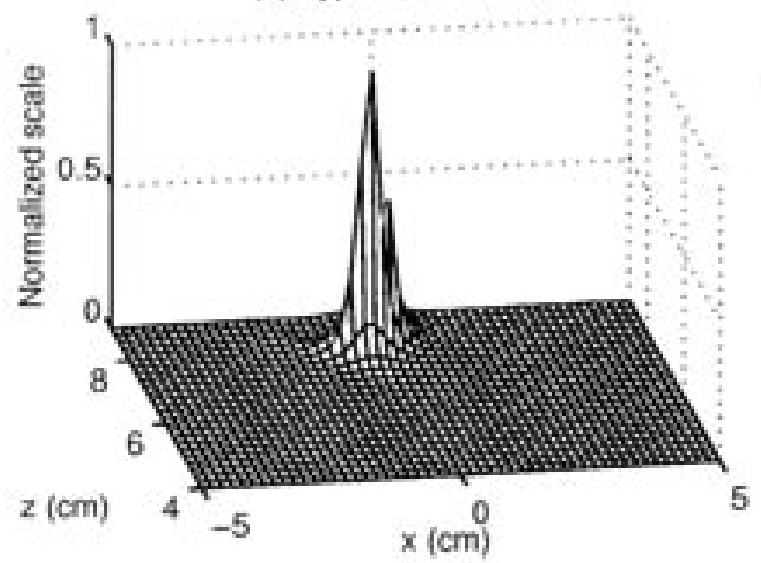

(c) Type 3 Beamformer

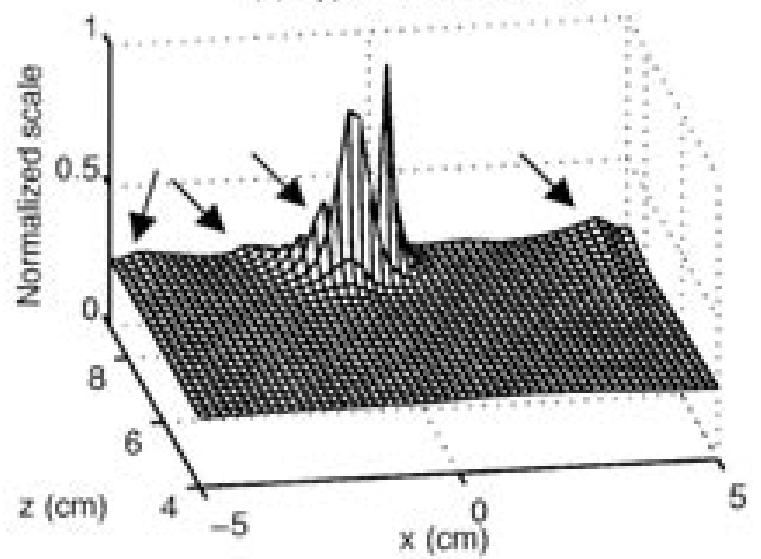

(b) Type 2 Beamformer

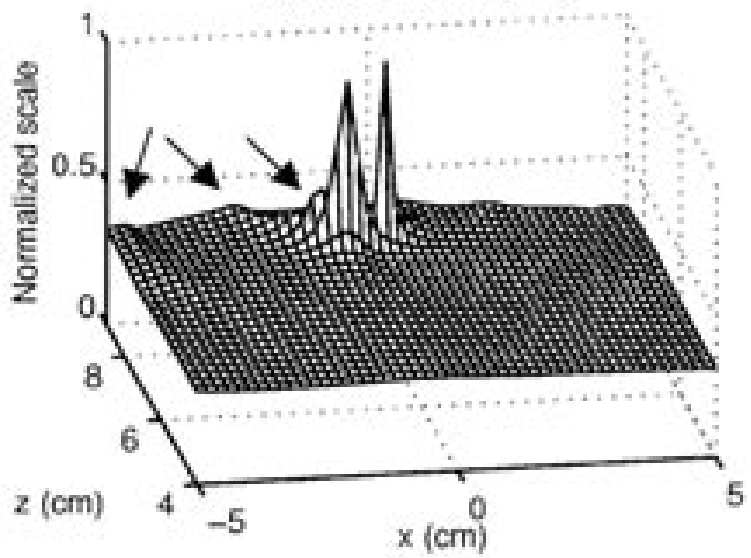

(d) Type 4 Beamformer

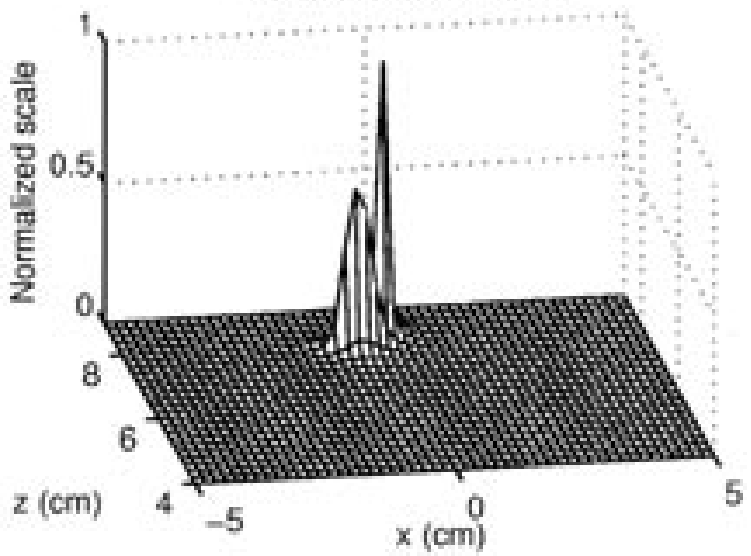

Figure 7. Normalized neuronal activity index for two $61 \%$ correlated dipole sources using 4 different vectorized beamformers at SNR=6. (a) The type 1 beamformer showed clean background and was able to separate two closely-spaced dipoles, but the separation was not very good. (b) Better separation was obtained from the type 2 beamformer, but additional smaller peaks were visible even at some distance from the real sources as indicated by arrows. (c) The type 3 beamformer also showed two major peaks and some additional smaller peaks either close to or far from the real sources. (d) Our type 4 beamformer clearly separated these two dipoles and with a clean background.

comparable to the type 2 and 3 beamformers. Furthermore, no visible false-positive activity was present using our beamformer, even in low SNR and high-correlation cases. In those cases, however, both type 2 and 3 beamformers showed additional local peaks in locations other than those of the simulated dipoles.

Substantial differences among the beamformers were also shown in the analyses of the MEG unilateral median-nerve stimulation data. The type 1 beamformer showed a clean background (low false-positive rate) for regions that were not supposed to be activated by this type of stimulation. However, weak activity was found in the areas that were expected to show strong neuronal responses (high false negative rate). In contrast, both the type 2 and 3 beamformers localized strong activity in many but not all of the areas that were predicted from neurophysiology data (low false negative rate), but considerable unexpected background activity was also present in both approaches (high false-positive rate). These results contrasted with our type 4 beamformer, in which we reliably obtained all expected areas of activation (low false negative rate) with low background activity (low false-positive rate). We note that the number of neuronal sources localized in the present study is substantially more than the previous EEG study by Van Drongelen and colleagues (1996) using the type 1 beamformer approach by Van Veen and colleagues (1997). In their EEG study, bilateral median-nerve stimuli with repetition rate of $7.2 \mathrm{~Hz}$ were used, and only bilateral SI sources were found. The difference in the number of sources lo- 


\section{(a) Type 1 Beamtormer}

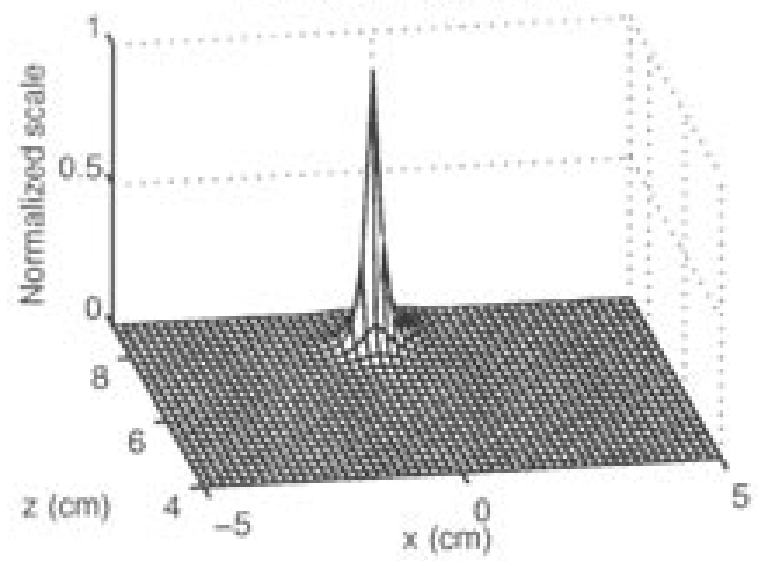

(c) Type 3 Beamformer

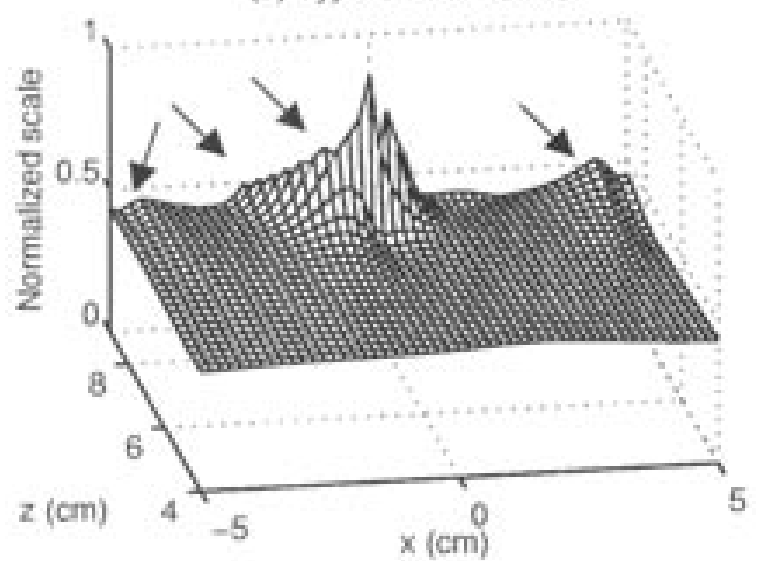

(b) Type 2 Beamformer

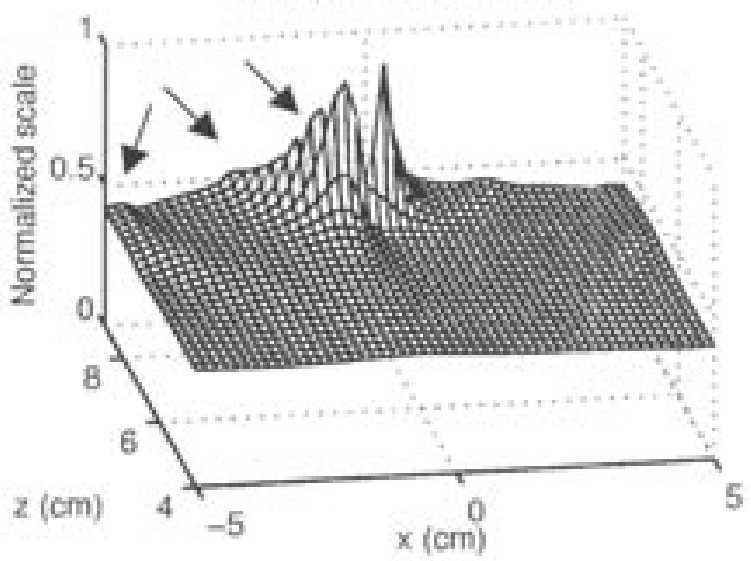

(d) Type 4 Beamformer

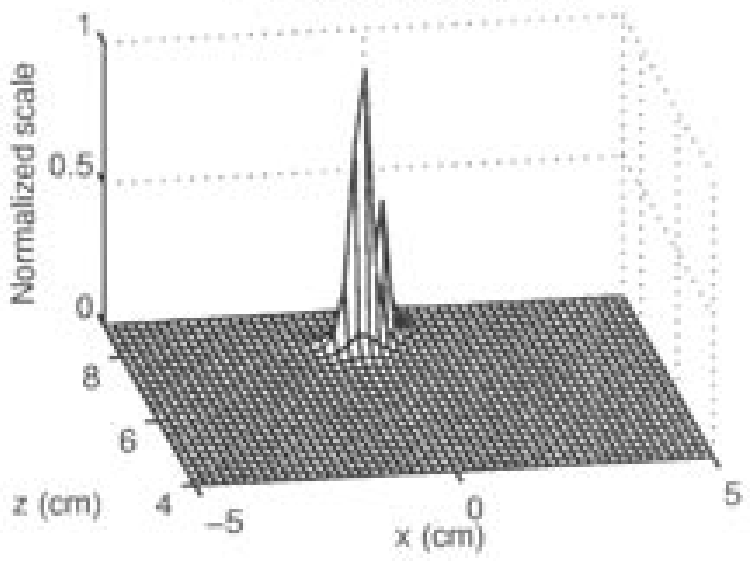

Figure 8. Normalized neuronal activity index for two $90 \%$ correlated dipole sources using 4 different beamformers at SNR=6. (a) The type 1 beamformer showed clean background and was unable to separate two highly-correlated and closely-spaced dipoles. (b) The type 2 beamformer separated the two dipoles, but there were many additional not-so-small peaks shown in the reconstruction. (c) Similar result was shown using the type 3 beamformer. (d) Our type 4 beamformer distinguished the two dipoles that were highly correlated, and the background remained clean with no visible false-positive activity.

cated in their study and the present work can be explained by at least three reasons: 1) The repetition rate used in Van Drongelen and colleagues' study is much higher than ours $(7.2 \mathrm{~Hz}$ versus $1 \mathrm{~Hz})$. At high simulation rate, sources with relative slow responses (e.g., SIIs, PrM, Prtl, and SMA) would not have the chance to reach their maximum levels when the following stimulus arrived. 2) EEG, instead of MEG, was used in their study. It is probable that the low conductivity of the skull tissue reduces EEG's ability to resolve neuronal sources that are spatially close whereas for MEG, the skull is virtually transparent. 3) The number of channels (64 channels) in their EEG study is much less than that of our MEG system (122 channels), which further reduces their ability to resolve the other neuronal generators.
A major reason why our beamformer performed better than other approaches is the use of a higher-order covariance matrix, which enhances the SNR. This enabled us to distinguish highly correlated sources that were difficult to resolve using the type 1 beamformer. " 11 illustrates why a higher-order covariance matrix improves the SNR. This figure shows the singular value decomposition (Golub and Van Loan 1983) results for the first-order (squares) and third-order (circles) covariance matrices from the two highly correlated sources $(90 \%$ correlation) used in the simulation with noise added. In this figure, singular values were normalized to the corresponding first (largest) singular value. Since the two sources were highly correlated, the second largest singular value from first-order covariance matrix was close to 


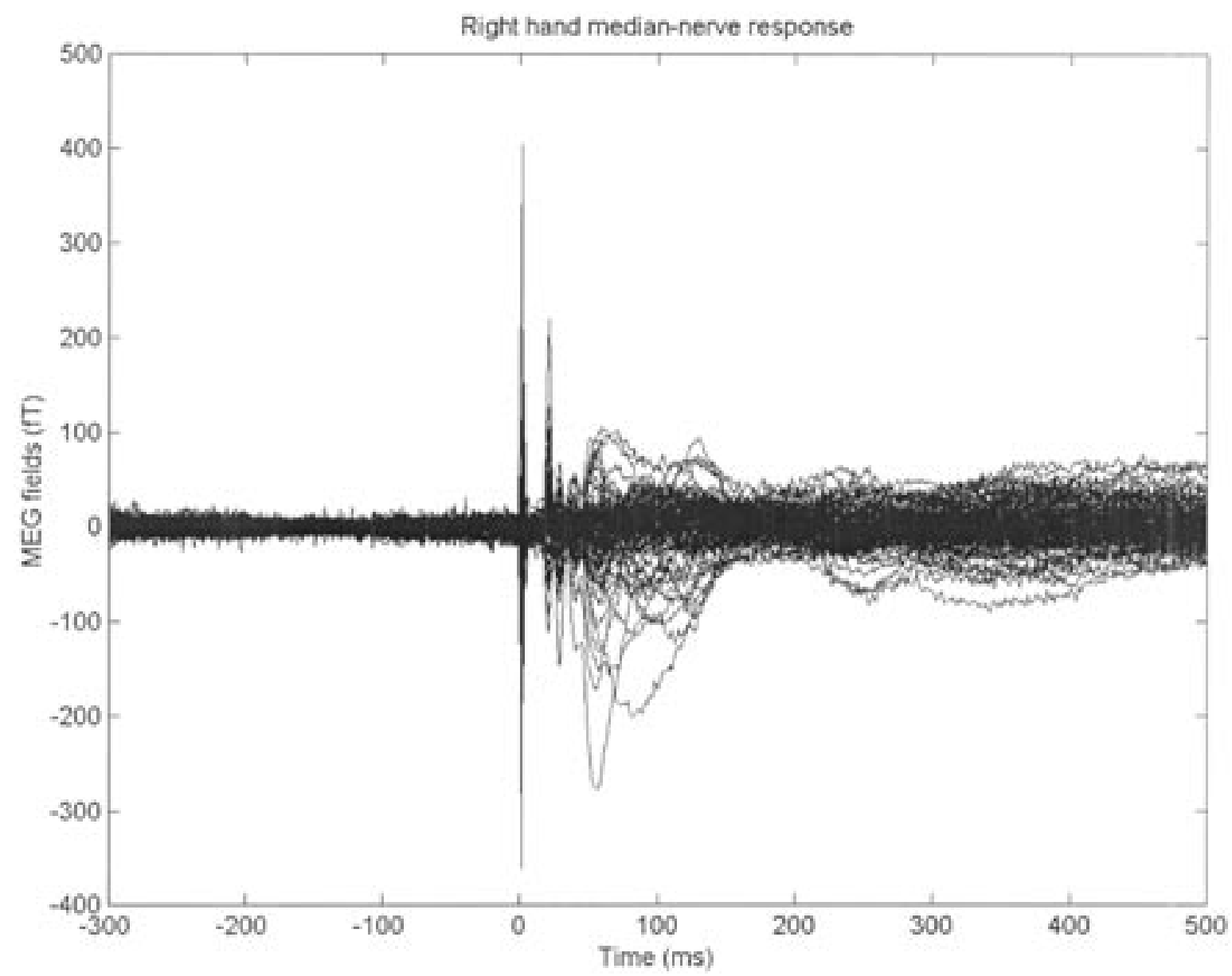

Figure 9. MEG sensor waveforms generated from right median-nerve stimulation in a healthy adult human volunteer. Waveforms from all 122 channels are superimposed.

the noise singular values, indicating that the temporal difference between the two correlated signals was approaching the noise level. However, for the singular value plot from the third-order covariance matrix, the second largest singular value was more than 50 times larger than the remaining noise singular values.

\section{Fixed- and Varied-Orientation Beamformer Approaches}

As mentioned previously, some developments of the beamformer require an extra step to optimize the dipole orientation for the entire time interval for which the signal covariance matrix is evaluated. This approach assumes that the source orientations do not change with time. Considering the situation that the time interval used for evaluating the signal covariance matrix is at least a few hundred milliseconds, the fixed-orientation assumption may not always be valid in dealing with empirical human MEG/EEG responses. In contrast, the vectorized beamformers described in the present study impose no constraint to the source orientations, so they are able to handle both fixed-orientation and varying-orientation situations. Another reason that some beamformers use the fixed-orientation assumption is the lack of theoretical formulas to merge beamformers from different source orientations into a single spatial index for each spatial location. In the present study, details were provided on how to merge beamformers from different source orientations into a single spatial index for each spatial location (equations 10, 12, 13, 16, and 18).

\section{Limitation of Covariance-Based Approaches}

In the present study, time windows containing a few hundred time points were used in analyzing both the simulations and the human median-nerve MEG responses with various beamformers. In contrast, it is common to use short time windows (even a single time point at the peak of the response) in modeling MEG/EEG 


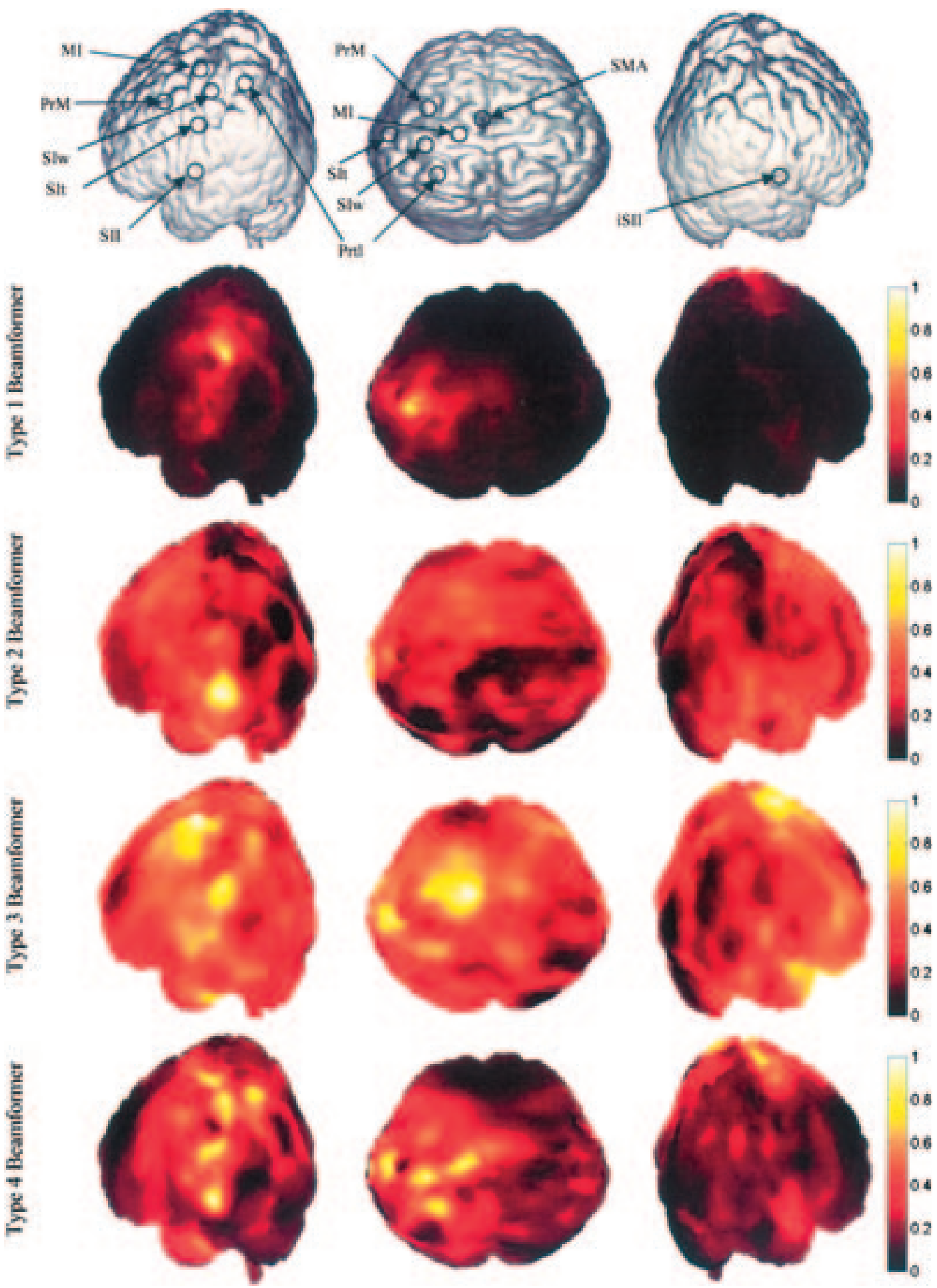

Figure 10. Results from analyses of median-nerve stimulation data using four beamformer approaches. Top row: left, superior, and right views of the cortical surface used in the calculation. Also shown are the expected cortical sources from neurophysiology literature: In primary somatosensory area for the wrist (SIw), primary somatosensory area for the thumb (SIt), primary motor area (MI), secondary somatosensory area (SII), premotor area (PrM), superior parietal area (Prtl), all in the left hemisphere contralateral to the side of the stimulation; plus the supplementary motor area (SMA) near the mid-line and the secondary somatosensory area in the right hemisphere ipsilateral to the stimulation (iSII). The neuronal activity index maps are displayed from the type 1 (second row), type 2 (third row), type 3 (fourth row), and our type 4 (fifth row) Beamformers. 


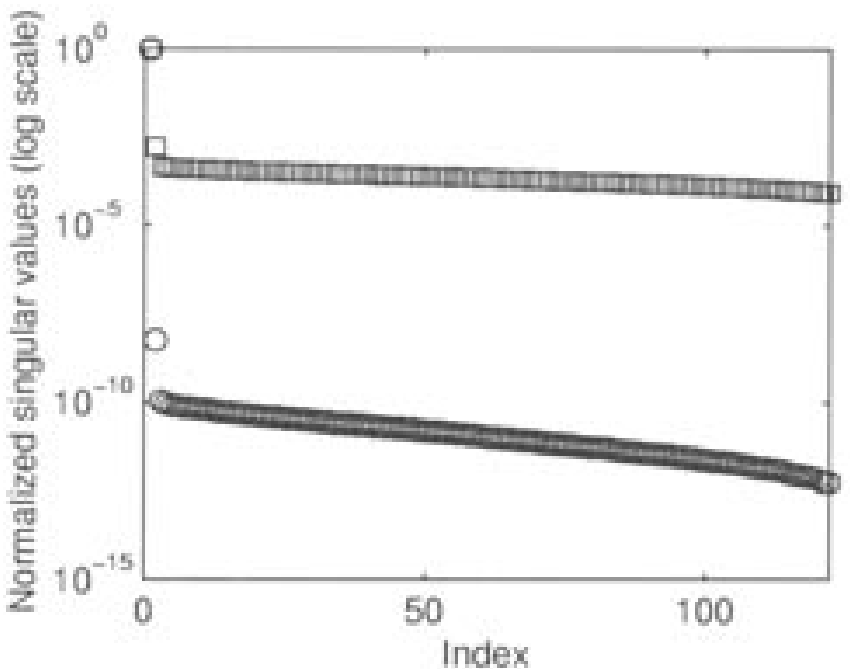

Figure 11. Singular value decomposition results for two different orders of the covariance matrices from the $90 \%$ correlated case in the simulation with noise (Fig. 8). Squares: singular-value plot of the first-order covariance matrix. Circles: singular-value plot of the third-order covariance matrix. The singular values were normalized to the corresponding first (largest) singular value. A logarithmic scale is used for the singular values.

sources using either dipole-fitting or minimum-norm procedures. However, as was pointed out by Van Veen et al. (1997), for covariance-based source localization techniques, such as beamformer, the number of time points must at minimum exceed the number of measurement channels for the interval used to calculate covariance matrices (both signal and noise covariance matrices), in order for the covariance matrices to be nonsingular. Furthermore, randomness in the covariance matrix will introduce randomness into the estimated spatial maps of brain activity. To reduce this randomness, a common rule of thumb is that the number of time points should be several times as large as the number of measurement channels (Van Veen et al. 1997). For this reason, it is not feasible for a beamformer (or other covariance-based source localization techniques) to fit smaller time windows, which is a limitation of all beamformer analyses.

\section{Conclusions}

Theoretical formulas were provided on all major aspects of existing EEG/MEG beamformers as well as a novel beamformer we developed that uses higher-order covariance analysis. We demonstrated that all existing EEG/MEG beamformers are minimum-variance beamformers, and their beamformer coefficients are identical if no regularization is applied. However, sub- stantial differences still exist among the different beamformer approaches in terms of the neuronal activity index. Computer simulations and empirical human median-nerve MEG data analysis showed that our beamformer has certain advantages over existing beamformers in terms of its high spatial resolution and low false-positive background activities, and its ability to deal with low SNR data, highly-correlated source time-courses, and large numbers of neuronal sources.

\section{References}

Baldissera, F. and Leocani, L. Afferent excitation of human motor cortex as revealed by enhancement of direct cortico-spinal action on motoneurones. Electroenceph. Clin. Neurophysiol., 1995, 97: 394-401.

Barba, C., Frot, M., Guenot, M. and Mauguiere, F. Stereotactic recordings of median nerve somatosensory-evoked potentials in the human pre-supplementary motor area. Eur. J. Neurosci., 2001, 13(2): 347-356.

Barnes, G.R. and Hillebrand, A. Statistical flattening of MEG beamformer images. Hum. Brain. Mapp., 2003, 18: 1-12.

Bergeron, J.W. and Braddom, R.L. Palmar cutaneous nerve recording and clarification of median premotor potential generators. Am. J. Phys. Med. Rehabil., 1998, 77(5): 399-406.

Boakye, M., Huckins, S.C., Szeverenyi, N.M., Taskey, B.I. and Hodge, C.J. Jr. Functional magnetic resonance imaging of somatosensory cortex activity produced by electrical stimulation of the median nerve or tactile stimulation of the index finger. J. Neurosurg., 2000, 93(5): 774-783.

Borgiotti, G. and Kaplan, L.J. Super resolution of uncorrelated interference sources by using adaptive array technique. IEEE Trans. Antennas Propagat., 1979, 27: 842-845.

Chang, L. and Yeh, C.C. Performance of DMI and eigenspace-based beamformers. IEEE Trans. Antennas Propagat., 1992, 40: 1336-1347.

Davidoff, R.A. The pyramidal tract. Neurology, 1990, 40: 332-339.

Forss, N., Hari, R., Salmelin, R., Ahonen, A., Hamalainen, M. Kajola, M., Knuutila, J. and Simola, J. Activation of the human posterior parietal cortex by median nerve stimulation. Exp. Brain. Res., 1994, 99(2): 309-315.

Forss, N. and Jousmaki, V. Sensorimotor integration in human primary and secondary somatosensory cortices. Brain Res., 1998, 781(1-2): 259-267.

Fujiwara, N., Imai, M., Nagamine, T., Mima, T., Oga, T., Takeshita, K., Toma, K. and Shibasaki, H. Second somatosensory area (SII) plays a significant role in selective somatosensory attention. Brain Res. Cogn. Brain Res., 2002, 14(3): 389-397.

Golub, G.H. and Van Loan, C.E. Matrix Computations. Baltimore, Maryland: The Johns Hopkins University Press, 1983.

Gross, J. and Ioannides, A.A. Linear transformations of data space in MEG. Phys. Med. Biol., 1999, 44: 2081-2097.

Gross, J., Kujala, J., Hamalainen, M., Timmermann, L., Schnitzler, A. and Salmelin, R. Dynamic imaging of coher- 
ent sources: studying neural interactions in the human brain. Proc. Natl. Acad. Sci. U.S.A., 2001, 98: 694-699.

Hari, R., Karhu, J., Hämäläinen, M., Knuutila, J., Salonen, O., Sams, M. and Vilkman, V. Functional organization of the human first and second somatosensory cortices: a neuromagnetic study. Eur. J. Neurosci., 1993, 5(6): 724-734.

Hari, R. and Forss, N. Magnetoencephalography in the study of human somatosensory cortical processing. Philos. Trans. R. Soc. Lond. B Biol. Sci., 1999, 354(1387): 1145-1154.

Huang, M., Aine, C.J., Supek, S., Best, E., Ranken, D. and Flynn, E.R. Multi-start downhill simplex method for spatio-temporal source localization in Magnetoencephalography. Electroenceph. Clin. Neurophysiol., 1998, 108: 32-44.

Huang, M., Aine, C., Davis, L., Butman, J., Christner, R., Weisend, W., Stephen, J., Meyer, J., Silveri, J., Herman, M. and Lee, R.R. Sources on the Anterior and Posterior Banks of the Central Sulcus Identified from Magnetic Somatosensory Evoked Responses using Multi-Start Spatio-Temporal Localization. Human Brain Mapping, 2000, 11: 59-76.

Jones, E.G., Coulter, J.D. and Hendry, S.H.C. Intracortical conectivity of architectonic field in somatic sensory, motor and parietalcortex of monkey. J. Comp. Neurol., 1978, 181: 291-348.

Jones, E.G., Wise, S.P. and Coulter, J.D. Differential thalamic relationships of sensory-motor and parietal cortical fields in monkeys. J. Comp. Neurol., 1979, 183: 833-892.

Jousmaki, V. and Forss, N. Effect of stimulus intensity on signals from human somatosensory cortices. Neuroreport, 1998, 9(15): 3427-3431.

Kawamura, T., Nakasato, N., Seki, K., Kanno, A., Fujita, S., Fujiwara, S. and Yoshimoto, T. Neuromagnetic evidence of pre- and post-central cortical sources of somatosensory evoked responses. Electroenceph. Clin. Neurophysiol., 1996, 100: 44-50.

Lemon, R.N. and Porter, R. Afferent input to movement-related precentral neurones in conscious monkey. Proc. R. Soc. Lond. [Biol]., 1976, 194: 313-339.

Lemon, R.N. and van der Burg, J. Short-latency peripheral inputs to thalamic neurones projecting to the motor cortex in the monkey. Exp. Brain. Res., 1979, 36: 445-462.

Lemon, R.N. Functional properties of monkey motor cortex neurones receiving afferent input from the hand and fingers. J. Physiol. (Lond)., 1981, 311: 497-519.

Mauguiere, F., Merlet, I., Forss, N., Vanni, S., Jousmaki, V., Adeleine, P. and Hari, R. Activation of a distributed somatosensory cortical network in the human brain: a dipole modeling study of magnetic fields evoked by median nerve stimulation. Part I: Location and activation timing of SEF sources. Electroenceph. Clin. Neurophysiol., 1997a, 104(4): 281-289.

Mauguiere, F., Merlet, I., Forss, N., Vanni, S., Jousmaki, V., Adeleine, P. and Hari, R. Activation of a distributed somatosensory cortical network in the human brain: a dipole modeling study of magnetic fields evoked by median nerve stimulation. Part II: Effect of stimulus rate, attention and stimulus detection. Electroenceph. Clin. Neurophysiol., 1997b, 104(4): 290-295.
McGlone, F., Kelly, E.F., Trulsson, M., Francis, S.T., Westling, G. and Bowtell, R. Functional neuroimaging studies of human somatosensory cortex. Behav. Brain Res., 2002, 135(1-2): 147-158.

Mosher, J.C., Lewis, P.S. and Leahy, R.M. Multiple dipole modeling and localization from spatio-temporal MEG data. IEEE Trans. Biomed. Eng., 1992, 39: 541-557.

Park, T.A. and Del Toro, D.R. Generators of the early and late median thenar premotor potentials. Muscle Nerve, 1995, 18(9): 1000-1008.

Robinson, S.E. and Vrba, J. Functional neuroimaging by syntheticaperture magnetometry (SAM). In: T. Yoshimoto, M. Kotani, S. Kuriki, H. Karibe and N. Nakasato (Ed.) Recent Advances in Biomagnetism, Tohoku University Press, Sendai, Japan, 1999:302-305.

Rosen, I. and Asanuma, H. Peripheral afferent inputs to the forelimb area of the monkey motor cortex: input-output relations. Exp. Brain Res., 1972, 14: 257-273.

Sarvas, J. Basic mathematical and electromagnetic concepts of the bio-magnetic inverse problems. Phys. Med. Biol., 1987, 32: 11-22.

Sekihara, K., Poeppel, D., Miyashita, Y. Application of eigenspace beamformer to virtual depth-electrode measurement using MEG. In S. Supek (Ed.) Proceedings 2nd International Symposium in Noninvasive Functional Source Imaging within the Human Brain and Heart (Biomedizinische Technik), Zagreb, Coratia, 1999:127-130.

Sekihara, K., Nagarajan, S.S., Poeppel, D., Marantz, A. and Miyashita, Y. Reconstructing spatio-temporal activities of neural sources using an MEG vector beamformer technique. IEEE Trans. Biomed. Eng., 2001, 48(7): 760-771.

Simoes, C., Jensen, O., Parkkonen, L. and Hari, R. Phase locking between human primary and secondary somatosensory cortices. Proc. Natl. Acad. Sci. U.S.A., 2003, 100(5): 2691-2694.

Spiegel, J., Tintera, J., Gawehn, J., Stoeter, P. and Treede, R.D. Functional MRI of human primary somatosensory and motor cortex during median nerve stimulation. Clin. Neurophysiol. 1999, 110(1): 47-52.

Urbano, A., Babiloni, F., Babiloni, C., Ambrosini, A., Onorati, P. and Rossini, P.M. Human short latency cortical responses to somatosensory stimulation. A high resolution EEG study. Neuroreport, 1997, 8(15): 3239-3243.

Uutela, K., Hamalainen, M. and Salmelin, R. Global optimization in the localization of neuromagnetic sources. IEEE Trans. Biomed. Eng., 1998, 45(6): 716-723.

Van Veen, B.D., van Drongelen, W., Yuchtman, M. and Suzuki, A. Localization of brain electrical activity via linearly constrained minimum variance spatial filtering. IEEE Trans. Biomed. Eng., 1997, 44(9): 867-880.

Van Veen, B.D. and Buckley, K.M. Beamforming: a versatile approach to spatial filtering. IEEE ASSP Mag. 1988: 4-24.

Vrba, J. and Robinson, S.E. SQUID sensor array configurations for magnetoencephalography applications. Supercond. Sci. Technol., 2002, 15: R51-R89.

Waberski, T.D., Gobbele, R., Darvas, F., Schmitz, S. and Buchner, H. Spatiotemporal imaging of electrical activity related to attention to somatosensory stimulation. Neuroimage, 2002, 17(3): 1347-1357. 
Wong, Y.C., Kwan, H.C., MacKay, W.A. and Murphy, J.T. Spatial organization of precentral cortex in awake primates. I. Somato-sensory inputs. J. Neurophysiol., 1978, 41: 1107-1120.
Wood, C.C., Cohen, D., Cuffin, B.N., Yarita, M. and Allison, T. Electrical sources in human somatosensory cortex: identification by combined magnetic and potential recording. Science, 1985, 227: 1051-1053. 